\title{
Employés publics dans les environnements hybrides : Quelles ancres d'engagement et quelles pratiques de GRH ?
}

\section{Public employees in hybrid organizational contexts: what commitment anchors and what HRM practices?}

Armand Brice Kouadio* et Yves Emery ${ }^{\dagger}$

\footnotetext{
* Armand Brice Kouadio, Assistant diplômé, Unité de management et gestion des ressources humaines, Institut de hautes études en administration publique, akouadio@unil.ch. T +41216926873.

$\dagger$ Yves Emery, Professeur ordinaire, Unité de management et gestion des ressources humaines, Institut de hautes études en administration publique.
} 


\title{
Résumé
}

Mobilisant une approche exploratoire, nous analysons les ancres d'engagement au travail chez les agents publics œuvrant dans des contextes post-bureaucratiques. La notion d'Engagement au travail élargit le concept d'engagement organisationnel (Organizational Commitment) à des conditions et réalités professionnelles non exclusivement liées aux organisations et présentant des caractéristiques hybrides. Cette recherche permet d'identifier quelles ancres d'engagement sont dominantes et typiques des environnements publics post-bureaucratiques, à côté des ancres classiques identifiées dans la littérature (organisation, équipe de travail, superviseur, emploi, profession), tout en analysant leurs implications en termes de GRH publique. En mobilisant un cadre analytique basé sur les ancrages multiples de la relation au travail, cette étude apporte une compréhension plus fine de l'engagement au travail des agents publics Suisses.

Mots clés : Engagement public au travail, ancres d'engagement, organisations publiques hybrides, GRH publique.

\begin{abstract}
Using an exploratory qualitative approach, we analyze the workplace commitment anchors of public officials working in post-bureaucratic contexts. The concept of public workplace commitment broadens organizational commitment to conditions and professional realities not exclusively related to organizations and bearing hybrid characteristics (Emery and Giauque 2014). This research identifies what commitment anchors are dominant and typical of post-bureaucratic public environments, alongside the traditional anchors described in the literature (organization, work team, supervisor, job, profession), and examine their implications in terms of public HRM. By mobilizing an analytical framework based on the multiple anchors of the relationship to work, it provides a deeper understanding of Swiss public employees' Workplace commitment.
\end{abstract}

Keywords: Public Workplace Commitment, commitment anchors, hybrid public sector organizations, public HRM 


\section{Contexte et problématique}

Marquées par d'importantes réformes d'inspiration gestionnaire dans les pays de I'OCDE (C. Hood, 1991; Christopher Hood \& Peters, 2004; Pollitt \& Bouckaert, 2009; Reichard, 2002), les organisations publiques actuelles tentent de concilier au mieux les valeurs et référentiels propres au service public avec ceux hérités du monde marchand (Boltanski \& Thévenot, 1991); lesquels mettent en avant notamment la performance et l'orientation «client». C'est ainsi qu'apparait progressivement la postbureaucratie (Christensen \& Laegreid, 2007; Emery \& Giauque, 2016; Pollitt \& Bouckaert, 2011), ce nouvel environnement hybride qui bouleverse les codes, valeurs et motivations au fondement des comportements adoptés par les agents publics. Les nombreuses études en la matière, notamment celles portant sur le PSM (Desmarais \& Gamassou, 2014), témoignent des questionnements profonds à cet égard. Si une littérature conséquente s'est interrogée sur les remises en cause identitaires et les nouveaux contours de l'ethos public (Buffat, 2014; I Fortier \& Emery 2012; Giauque, Ritz, Varone, \& Anderfuhren-Biget, 2012; Horton, 2006), une autre, plus conséquente encore, a porté sur les valeurs et motivations spécifiques aux personnels publics, qui s'inscriraient en faux par rapport aux logiques dominantes des réformes managériales (Giauque, Anderfuhren-Biget, \& Varone, 2013; J.L. Perry, 1996; W. Vandenabeele, Steijn, Camilleri, \& et Al, 2008). À l'inverse, très peu d'études récentes se sont penchées sur l'engagement des agents publics, et lorsqu'elles l'ont fait, elles sont restées cantonnées à l'engagement organisationnel, dans la tradition des recherches basées sur le concept tridimensionnel développé par Allen et Meyer (J. P. Meyer, D.J. Stanley, L. Herscovitch, \& L. Topolnytsky, 2002a). Or, il est important de proposer des modèles permettant de caractériser plus finement la spécificité de l'engagement au travail dans le secteur public (G. A. Boyne, 2002; Buchanan, 1974; Nyhan, 1999), particulièrement dans un environnement désormais hybride impliquant des valeurs et principes de gestion autant publics que privés. Ceci à une époque où les trajectoires de carrière deviennent de plus en plus nomades, voire "protéiformes" (Franck Biétry \& Laroche, 2011; D. Hall, 1976). II paraît donc théoriquement approprié et empiriquement pertinent d'étendre le concept d'engagement organisationnel audelà des frontières de ces organisations précisément. Tout en reconnaissant l'effet modérateur des réformes NGP, la plupart des études de l'engagement organisationnel des agents publics dans les environnement post-bureaucratiques (Steijn \& Leisink, 2006) se sont surtout penchées sur ses antécédents (Castaing, 2006; Giauque, Resenterra, \& Siggen, 2013; Jung \& Ritz, 2014; Rayner, 2012). Ces études révèlent des déterminants spécifiques au secteur public tels que le soutien politique aux réformes, 
Revue de Gestion et Management Public

notamment chez les managers (Oh \& Park, 2011; Yang \& Pandey, 2009); le PSM (Camilleri, 2006; Markovits, Davis, Fay, \& Dick, 2010; J.L. Perry, 1996), I'habilitation psychologique (Vandenberghe, 2005), et l'adéquation avec les buts et valeurs portés par les réformes du service public en cours (Giauque, Resenterra, et al., 2013). Pour autant, ces antécédents de l'engagement organisationnel n'apportent aucun éclairage particulier sur des ancres nouvelles susceptibles de mobiliser les agents publics au XXlème siècle. Dans le sillage des théoriciens ayant conceptualisé les engagements multiples, plusieurs auteurs recommandent désormais d'intégrer l'autre versant de la multi-dimensionnalité du concept d'engagement organisationnel : ses ancres, et partant les comportements discrétionnaires qui s'y rapportent (T. E. Becker, Billings, Eveleth, \& Gilbert, 1996; Van Dick, Wagner, Stellmacher, \& Christ, 2004). Ainsi donc, la rareté des travaux consacrés aux environnements de travail post-bureaucratiques, lesquels voient tendanciellement une altération de la relation de travail et du lien individu-organisation (Diefenbach, 2009), justifie à notre sens le besoin d'une conceptualisation nouvelle de l'engagement au travail des agents publics.

\section{Objectifs et questions de recherche}

L'engagement désigne une force liant l'individu à une action pertinente en rapport avec une ancre donnée, et se traduisant par une attitude ou un comportement visant à maintenir ce lien (J.P. Meyer \& L. Herscovitch, 2001). Ainsi est-elle susceptible de porter sur des ancres diverses: I'organisation (Mathieu \& Zajac, 1990; John P. Meyer \& Allen, 1991; R.T. Mowday, Porter, \& Steers, 1982), le syndicat (Barling, Wade, \& Fullagar, 1990; Gordon, Philpot, Burt, Thompson, \& Spiller, 1980), I'emploi et la profession (Blau, 1985; John P. Meyer, Natalie J. Allen, \& Catherine A. Smith, 1993), l'équipe et le superviseur (Thomas E. Becker, 1992; Hunt \& Morgan, 1994; Locke, Latham, \& Erez, 1988), la carrière etc. (D. T. Hall \& al, 1996). Alors que la littérature peine à s'accorder sur la différence de niveau d'engagement entre personnels des secteurs public et privé, et sur la spécificité de l'engagement organisationnel public (G. A. Boyne, 2002; Goulet \& Frank, 2002), nous proposons ici une conception plus large de l'engagement, à travers le concept d'« engagement public au travail » (PWPC ou Public Workplace Commitment en anglais) afin d'en explorer et découvrir les ancres dominantes. Le PWPC désigne dans le cadre de cet article, La manière dont les employés publics s'identifient, s'attachent, agissent et expriment leur loyauté, parfois au-delà de leurs propres intérêts, à des buts, valeurs et attitudes incarnées 
Revue de Gestion et Management Public

dans des ancres plus ou moins saillantes au sein de leurs environnements de travail (inspiré de (Valéau, 2007; W. Vandenabeele et al., 2008).

Sur cette base conceptuelle étendue, les objectifs de la présente recherche sont résumés ainsi :

1. Interroger les ancres d'engagement au travail des agents publics œuvrant dans des environnements plus ou moins hybrides (comme c'est le cas pour la fonction publique suisse, d'une manière générale cf. Emery, 2013).

2. Cartographier les ancres dominantes dans lesquelles les agents publics trouvent le sens de leurs engagements au travail, et en discuter la publicitude, à savoir en quoi ces ancres revêtent une dimension publique ou non.

3. Analyser les implications de ces ancres d'engagement en matière de gestion du capital humain dans le secteur public.

Dans les lignes qui suivent, nous revenons d'abord sur l'engagement au travail comme concept majeur de la relation employé-employeur. Fondant notre argumentation sur les limites et les voies laissées inexplorées par cette recherche dans le secteur public, nous montrons comment la multi dimensionnalité de l'engagement se traduit également par la multiplicité de ses ancres. Suite à la description de notre méthodologie, qualitative-exploratoire, nous présentons nos résultats dont nous discutons les implications en terme de GRH publique.

\section{L'engagement organisationnel des agents dans le secteur public}

\subsection{Origines du concept d'engagement organisationnel}

Le concept d'engagement organisationnel est né au début des années 1960 grâce aux travaux de Becker sur les paris subsidiaires (H. S. Becker, 1960), lesquels impliquent une série de décisions individuelles cohérentes. Appliquée au monde du travail, la théorie des paris subsidiaires permet d'expliquer notamment la loyauté organisationnelle. Dans ces années d'après-guerre, la forte pénurie de main d'œuvre qualifiée, et le besoin de relance économique dans un contexte de forte compétitivité, orientèrent l'intérêt pour la sécurisation et le maintien des ressources (humaines) dans les organisations. En continuité des théories organisationnelles mettant l'emphase sur les relations humaines (Likert, 1961, 1967; Mayo, 1933; McGregor, 1960), les chercheurs désiraient avant tout comprendre comment engager les cœurs et les esprits, et non uniquement les bras. La popularité de la recherche sur 
Revue de Gestion et Management Public

l'engagement organisationnel s'explique ensuite par ses liens avec la fidélisation et la performance organisationnelles, mais également par sa force explicative des attitudes et des comportements organisationnels. Malgré la grande complexité de ce concept, manifeste dans le débat autour de son caractère attitudinal ou comportemental ( $F$. Biétry, Creusier, Laroche, \& Camus, 2014; Franck Biétry \& Laroche, 2011; Riketta, Van Dick, \& Rousseau, 2006), ou même sur sa dimensionnalité multiple, la domination du modèle tridimensionnel d'Allen et Meyer (Allen \& Meyer, 1990; John P. Meyer \& Allen, 1991; J. P. Meyer, N. J. Allen, \& C. A. Smith, 1993; J. P. Meyer, D. J. Stanley, L. Herscovitch, \& L. Topolnytsky, 2002b) reste aujourd'hui acquise. Selon ce modèle, l'engagement organisationnel repose sur les trois piliers qui sont l'engagement affectif $(A O C)$, l'engagement normatif (NOC), et l'engagement calculé (COC). Ce dernier modèle n'a cependant rien de spécifiquement public et s'ancre principalement dans l'organisation, d'où la question de recherche sous-jacente à la présente contribution: qu'en est-il réellement dans les organisations publiques hybrides (abritant à la fois valeurs et principes de management privés comme publics) telles que celle que nous en trouvons en Suisse, mais également dans de nombreux autres pays (Giauque / Emery, 2016) ?

\subsection{Comparaison de l'engagement organisationnel chez les personnels publics et privés}

Les travaux comparant l'engagement organisationnel dans les secteurs public et privé datent des années 1970s et du début des années 1980s. Ils coïncident donc pour la plupart avec l'émergence des réformes managériales dans le secteur public. Leurs résultats apparaissent plutôt contrastés : en somme, les antécédents de l'engagement organisationnel chez les agents publics se retrouvent pour l'essentiel dans les principales catégories (individuelles, organisationnelles, liées à l'emploi) identifiées dans la littérature, à l'exception de la Motivation de service public (PSM) et du Contrat Psychologique (CP) (J. A. Coyle-Shapiro \& Neuman, 2004; Mathieu \& Zajac, 1990; J. P. Meyer et al., 2002b; Moon, 2000a). D'autre part on peut constater l'idée prépondérante que les managers publics, ou les employés de façon générale, sont moins motivés, moins loyaux, avec une plus faible identification organisationnelle (Goulet \& Frank, 2002; Moon, 2000b; Odom, Boxx, \& Dunn, 1990; Hal G Rainey, 1989; Savery, 1991; Zeffane, 1994). Divers arguments viennent à l'appui de cette différence : pour Bourantas, cela est dû au décalage entre la culture espérée et la culture perçue dans les organisations publiques (Bourantas, Anagnostelis, Mantes, \& Kefalas, 1990). 
Revue de Gestion et Management Public

Si cette différence culturelle est reconnue par Cho et Lee dans leur échantillon d'agents publics Coréens de niveau managérial, elle ne suffit pas, selon les auteurs, à expliquer une éventuelle différence de niveau d'engagement (Cho \& Lee, 2001). Goulet et Frank (1995) mettaient quelques années auparavant plutôt le niveau moindre d'engagement des employés publics sur le compte d'un moindre recours aux récompenses extrinsèques, du fait de certains principes légaux liés à l'égalité de traitement et à l'équité. Cette explication est cependant rejetée par les auteurs pour qui le plus important dans l'engagement des agents publics est à rechercher dans la satisfaction de leurs besoins intrinsèques (J. A. M. Coyle-Shapiro \& Kessler, 2003). La méta-analyse de 44 études empiriques conduite par Boyne (2002) conclut que le moindre engagement organisationnel dans le public est lié à des procédures et une bureaucratie inflexibles, et au caractère indirect de la relation entre les performances au travail et les récompenses afférentes. Il fait néanmoins le constat suivant : les trois cinquièmes $(3 / 5)$ des études analysées révèlent des niveaux d'engagement plus élevés dans le privé, alors que le reste $(2 / 5)$ conclut que les agents publics sont les plus engagés, ou même qu'il est difficile de trancher la question en termes de niveaux d'engagement (G. A. Boyne, 2002). Ces résultats font écho aux observations formulées dès les années 1990, conduisant à relativiser la dimension organisationnelle de l'engagement au travail dans le secteur public (Danny $L$ Balfour \& Wechsler, 1990; Danny L. Balfour \& Wechsler, 1991, 1996). D'où l'intérêt de la présente recherche qui cherche à examiner la possibilité d'autres ancrages à l'engagement au travail chez les employés publics.

En résumé, la littérature n'a pas encore résolu la question de savoir si la différence d'engagement entre employés publics et privés s'exprime en niveau, ou à contrario, par rapport à son sens ou sa nature. Ceci en particulier dans des environnements managérialisés ou post-bureaucratiques du secteur public (Danny L Balfour \& Wechsler, 1990; Clugston, Howell, \& Dorfman, 2000).

\section{Engagement organisationnel dans les environnements post- bureaucratiques}

\subsection{L'engagement au travail dans le public : un engagement multi-ancres}

La définition classique de l'engagement organisationnel, basée sur la loyauté organisationnelle, dépeint bien la situation au début des années 1980 : une époque 
au cours de laquelle l'essentiel de la vie professionnelle se déroulait bien souvent dans une seule organisation. Ainsi, on "faisait carrière " chez son employeur. Cela s'applique cependant difficilement à la situation d'aujourd'hui, caractérisée par des carrières de plus en plus nomades et une évolution des attentes envers le travail ( $F$. Biétry et al., 2014). En se référant à la littérature traitant des ancres universelles d'engagement au travail, force est de constater que plusieurs études démontrent que l'engagement public présente des caractéristiques non nécessairement en lien avec l'organisation. C'est ainsi que sont apparues dans la recherche des études portant sur d'autres ancres d'engagement telles que le superviseur (T. E. Becker et al., 1996), la carrière (Ellemers, de Gilder, \& van den Heuvel, 1998), ou l'emploi (John P. Meyer et al., 1993). Certains travaux n'hésitent pas à considérer les entités régionales d'organisations géographiquement dispersées parmi les ancres possibles de l'engagement au travail (Scott, 1999). Pour ce qui est des ancrages tels que le travail, l'équipe, la carrière, le superviseur et l'organisation, ils sont aujourd'hui considérées comme universels ou génériques dans l'étude de l'engagement au travail (Thomas $E$. Becker, 1992; Aaron Cohen, 1999; Fornes \& Rocco, 2013; Morrow \& Wirth, 1989). Or pour la plupart, ces études ont souvent porté sur des entreprises privées, si ce n'est sur des populations de managers (Danny L Balfour \& Wechsler, 1990; Cho \& Lee, 2001; Zeffane, 1994) dont on sait qu'ils présentent des niveaux d'engagement bien souvent plus élevés que ceux des employés subalternes (Mathieu \& Zajac, 1990; J. P. Meyer et al., 2002b; Riketta, 2002).

\subsection{Les ancres d'engagement au travail : une dynamique à découvrir}

L'organisation n'étant pas un bloc monolithique, la conceptualisation d'un engagement multiple, focalisé sur différents sous-groupes, ou constituantes de l'organisation, s'impose d'elle-même (A. Cohen, 2006; Morrow \& Wirth, 1989; Reichers, 1985). La recherche définit l'ancre d'engagement comme susceptible d'être une entité (organisation, unité de travail etc.), une idée abstraite (une politique) ou le résultat d'une action telle que l'atteinte d'un objectif (Thomas E. Becker, 1992; Bishop \& Dow Scott, 2000; John P Meyer \& Lynne Herscovitch, 2001; Siders, George, \& Dharwadkar, 2001). Étant donnée la pluralité des référentiels axiologiques existant dans les organisations publiques contemporaines - en raison notamment de leur hybridation culturelle - (Y. Emery \& Giauque, 2014), il est probable qu'un nombre plus important d'ancres nouvelles puissent être mises en exergue par la présente recherche. D'autre part se pose la question de la hiérarchisation et de l'importance relative de certaines ancres d'engagement. La théorie des champs de Lewin (1943), et 
Revue de Gestion et Management Public

plus tard celle des engagements emboîtés de Lawler (1992), offrent un cadre d'analyse du dynamisme inhérent à ces ancres. La première, basée sur l'idée de la proximité psychologique, examine comment certains facteurs produisent des effets plus importants sur les comportements individuels (Lawler, 1992; Kurt Lewin, 1943). La seconde explique le développement de l'engagement au travail chez les individus " nichés » dans différents sous-groupes organisationnels (Lawler, 1992). Derrière ces deux théories, manifestement complémentaires, se cache l'idée selon laquelle l'ancre la plus saillante psychologiquement est celle vers laquelle s'oriente le comportement discrétionnaire (Stets \& Burke, 2000). C'est ainsi que les auteurs s'inscrivant dans ce courant se sont d'abord intéressés aux engagements " duals", en particulier envers l'organisation et le superviseur pour en examiner les rapports conflictuels (Thomas $\mathrm{E}$. Becker, 1992; Bentein, Vandenberghe, \& Dulac, 2004; Siders et al., 2001). Ces auteurs sont parvenus à la conclusion que le superviseur, en tant qu'ancre proximale ou distale, explique mieux la performance au travail. Cependant, qu'elles soient proximales, locales ou latentes, les multiples ancres d'engagement au travail ne s'excluent pas mutuellement, et reflètent simplement toute la complexité et la diversité des relations employé-employeurs (J.-W. Kim \& Rowley, 2005).

La prise en compte d'ancres multiples d'engagement dans le secteur public suppose la reconnaissance de formes individuelles et empathiques d'investissement au travail, à côté du lien avec l'organisation à proprement parler (Paillé, 2009 ; Cohen, 2006, Stinglhamber et Vandenberghe, 2003). Puisqu'il s'agit ici de faire ressortir les ancres des agents publics, la question de la publicitude de ces dernières se pose également. En d'autres termes, I'hybridité constatée dans les organisations publiques contemporaines, notablement en Suisse, se reflète-elle dans les ancres d'engagement que nous comptons identifier?

En résumé, il apparaît que l'état de la connaissance demeure lacunaire tant sur le plan des ancres pertinentes dans les contextes post-bureaucratiques, qu'en ce qui concerne leurs interactions dynamiques et leur caractère public. La méthode exploratoire développée dans la présente étude apporte un éclairage à la question des ancres dominantes de l'engagement au travail dans le secteur public, ouvrant ainsi la voie à une contextualisation de ce concept aux environnements de travail postbureaucratiques. 


\section{Méthodologie}

Cette recherche se fonde sur un design qualitatif exploratoire étant donnée la rareté de telles approches dans les études de l'engagement au travail dans les organisations publiques et parapubliques. Elle repose également sur une posture constructiviste et interprétative qui reconnaît que les individus, tout en étant surdéterminés par les cadres socio-professionnels dans lesquels ils évoluent, les transforment également, selon leurs perceptions des multiples interactions vécues au sein de ces organisations ${ }^{3}$ (Creswell, 2008). Un tel prisme permet, en ouvrant le champ des possibles, de favoriser l'émergence d'un nouveau concept, celui d'engagement public au travail PWPC - appréhendé ici à partir de ses ancres (Miles, Huberman, \& Saldana, 2013).

\subsection{Profil des répondants et déroulement des entretiens}

Nous avons rencontré, dans le cadre de cette recherche au total 29 employés du secteur public. Les répondants sont des femmes ou des hommes non cadres, avec une ancienneté moyenne de dix ans (Min=3 ans; Max=30 ans). Ils occupent des fonctions génériques non spécifiquement publiques (en l'occurrence de support) ou, au contraire, spécifiquement publiques, dans des environnements de travail divers du secteur public. Ces personnes sont en outre employées dans l'administration publique centrale au niveau communal, un établissement de santé pour personnes âgées, un centre de recherche universitaire, et une entreprise de transport public etc. Âgées entre 25 et 59 ans $(M=42)$, nos répondants présentent le profil adéquat pour cette étude, dans laquelle nous souhaitions recruter des individus susceptibles d'avoir un certain recul sur leur expérience d'employé public. On remarque une légère surreprésentation de femmes (17/29), avec près de la moitié des individus occupant des postes génériques (12/29), souvent des fonctions de support dans les Ressources Humaines, les finances ou la planification.

Sans annoncer explicitement l'objet de notre étude, nous avons précisé aux répondants les conditions dans lesquelles se dérouleraient les entretiens, leur caractère anonyme, les usages potentiels du corpus recueilli, ainsi que la possibilité d'y avoir accès, y compris au produit final de la recherche. Les entretiens ont été

\footnotetext{
${ }^{3}$ Dans le sens du Verstehen de Weber pour qui l'approche interprétative désigne une reconstruction réflexive et une interprétation des actions des autres, de sorte à ce que le sens émerge des intentions et des buts de l'individu.
} 
Revue de Gestion et Management Public

conduits à l'aide d'un guide avec des questions s'articulant autour du triptyque identification, attachement et loyauté. Ces thèmes se retrouvent majoritairement dans les principales échelles de mesure de l'engagement organisationnel (John P. Meyer \& Allen, 1991; Richard T. Mowday, Steers, \& Porter, 1979). Tout le long de ces entretiens nous avons fait l'effort d'adopter une attitude d'ouverture, en cohérence avec la dimension exploratoire de l'étude. Ci-après des extraits du guide d'entretien, illustratifs de chacun des trois axes:

- Identification: À quoi vous identifiez-vous le plus dans votre travail ? Qu'estce qui vous définit le mieux professionnellement?

- Attachement: À quoi êtes-vous le plus attaché(e) ? Seriez-vous prêt à quitter votre emploi actuel si ce facteur n'existait pas (l'objet d'attachement)?

- Loyauté: Qu'est ce qui a le plus d'impact sur votre volonté de continuer à travailler ici ou dans le secteur public ? Pourquoi ?

Les entretiens, d'une heure en moyenne (entre 45 minutes et $1 \mathrm{~h} 20$ ), ont tous été enregistrés. L'identification des ancres d'engagement public autorisait une retranscription sélective, consistant à ne saisir que les extraits d'intérêt, ainsi que des citations potentielles à utiliser pour illustrer certains arguments de la présente contribution.

\subsection{Techniques d'analyse}

À l'aide de l'outil d'analyse qualitative $\mathrm{NVIVO}^{4}$, une première série de codages reprenant les termes cités par les répondants comme ancres d'engagement a été effectuée. Dans une deuxième phase, les entretiens ont été relus afin de faire émerger des ancres (= nœuds selon le vocabulaire Nvivo) supplémentaires. Le mot, la phrase ou le paragraphe ont été utilisés comme unités de sens (et de codage). La phase de codage a aussi mobilisé nos notes d'entretien. Le corpus de nœuds ainsi formé, sur le mode du codage ouvert (Strauss \& Corbin, 1998), compte plus d'une centaine d'items que nous avons ensuite thématisés. Cette procédure, qui rappelle le codage axial recommandé dans la recherche qualitative liée à la théorie enracinée (Gioia, Corley, \& Hamilton, 2013; Strauss \& Corbin, 1998), a permis de dégager des regroupements thématiques autour des ancres principales d'engagement au travail. Le résultat de

\footnotetext{
${ }^{4}$ NVivo qualitative data analysis Software; QSR International Pty Ltd. Version 10, 2012.
} 
Revue de Gestion et Management Public

notre analyse est représenté dans la Figure 1 ci-dessous que nous commentons dans la partie suivante.

Figure 1 : L'engagement public au travail : un ancrage tridimensionnel

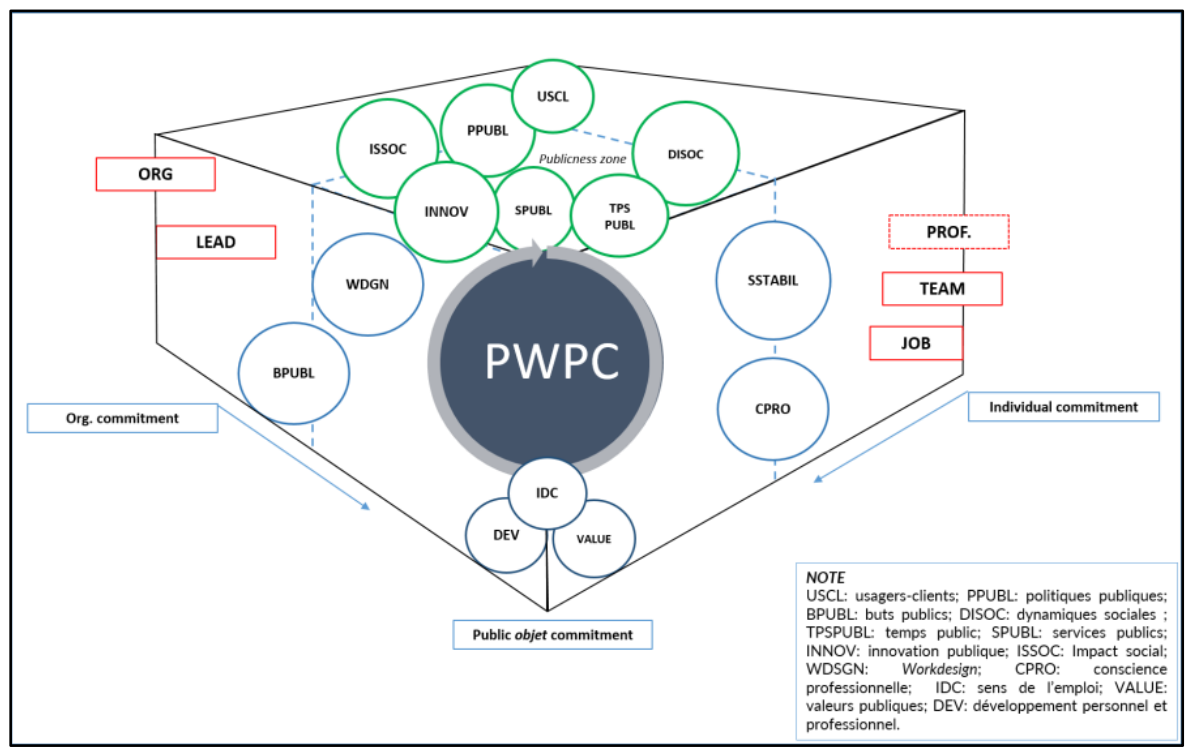

\section{Résultats et analyse}

Nos analyses révèlent plusieurs facettes de l'engagement au travail, représentant différents niveaux possibles d'ancrage de l'engagement au travail chez les employés publics interviewés. Ces facettes multiples de l'engagement au travail peuvent être organisées en grappes qui en révèlent les dimensions individuelles (CIND), organisationnelles (CORG) et "publiques" (CPUBL), soit un espace tridimensionnel d'engagement au travail (Figure 1). Toutefois, des chevauchements restent possibles entre ces catégories. La zone de publicitude, au cœur de cet espace tridimensionnel d'engagement au travail, croise à la fois les ancres s'éloignant des axes individuel et organisationnel, ainsi que celles ne rentrant pas dans ces deux catégories. Dans cet espace, mobilisé ici comme hypothèse de travail, les ancres publiques d'engagement au travail transcendent à la fois l'individualité de l'employé et le niveau organisationnel, comme le montrent les flèches de la Figure 1. 
Revue de Gestion et Management Public

Ainsi donc, l'engagement au travail dans les environnements publics sondés repose sur des ancrages multiples, plus ou moins individuels, organisationnels et publics, que nous commentons ci-après.

\subsection{L'engagement au travail dans le secteur public : des ancrages multiples}

Les ancres d'engagement au travail, révélées par regroupement successifs et comparaison jusqu'à l'obtention de catégories relativement exclusives les unes des autres font l'objet d'une définition et d'une illustration dans le Tableau de l'annexe 2. Nous y mettons en exergue les apports de cette étude en utilisant un code couleur. Ainsi, les cases vertes désignent les ancres d'engagement au travail ne faisant pas partie des cinq ancres classiques (en rouge) que sont l'Organisation, le Superviseur, I'Équipe de travail, l'Emploi, et la Carrière/Profession ${ }^{5}$. Ces ancres recèlent plus ou moins une spécificité publique, comme le laissent deviner leurs appellations de Service public (SPUBL), Usagers de l'action publique (USCL), Politiques publiques (PPUBL), Temps public (TPUBL) etc. Cette spécificité publique mériterait d'être testée. Nous utilisons donc ici le terme "ancres publiques" par souci de simplification. D'ailleurs, l'objectif de la présente étude est d'explorer l'existence d'ancres autres extra-organisationnelles, en particulier les plus dominantes dans les environnements de travail étudiés. Une seule ancre parmi toutes celles découvertes, en l'occurrence le Développement professionnel et personnel (DEV), est marquée d'un code couleur orange, étant donné qu'on peut difficilement la classer parmi les ancres classiques d'engagement au travail, encore moins parmi celles à priori publiques.

Selon le modèle proposé par Fornes et Rocco (2013), il est encore possible de subdiviser les ancres classiques d'engagement au travail en deux principaux sousgroupes : d'abord un engagement individuel, manifeste chez l'employé par la force de son identification avec les valeurs de ses pairs (collègues ou TEAM), ainsi que son emploi (JOB) et sa carrière (CAREER). Ensuite, l'engagement organisationnel à proprement parler regroupant l'engagement envers l'organisation elle-même (ORG), et l'engagement envers le superviseur (LEAD) : le superviseur étant considéré dans ce dernier cas comme une représentation symbolique de l'organisation. En complément

\footnotetext{
${ }^{5}$ Ici, Organisation $=$ ORG, Superviseur $=$ LEAD, Équipe de travail $=$ TEAM, Emploi $=$ JOB, Carrière/Profession $=$ PROF.
} 
Revue de Gestion et Management Public

de cette typologie, notre analyse des verbatim permet de révéler, comme évoqué supra, d'autres ancres, potentiellement publiques.

Fort de ces résultats, nous pouvons également de dégager des grappes d'ancres d'engagement au travail suivant une logique autre que celle proposée par Fornes et Rocco (2013).

\subsection{Clusters possibles d'ancres d'engagement au travail}

En reconsidérant les dimensions individuelle, organisationnelle et publique de l'engagement au travail (PWPC ou Public Workplace Commitment), quatre clusters $\mathrm{d}^{\prime}$ ancres sont identifiables : le premier en rouge sur la Figure 1, se compose des ancres organisationnelles et individuelles, que l'on retrouve parmi les ancres universelles d'engagement (JOB, TEAM, LEAD, PROF et ORG). Le second cluster rassemble deux ancres d'engagement à mi-chemin entre les niveaux individuel et public, en l'occurrence la Conscience professionnelle (CPRO) et la Sécurité et la stabilité de l'emploi (SSTABIL), ainsi que deux ancres recoupant les niveaux organisationnels et publics : le Workdesign et les Buts publics (respectivement WDGN, et BUTS). La particularité de ce dernier cluster est celle d'être composé d'ancres d'engagement à cheval sur deux dimensions différentes. Ainsi la Conscience professionnelle représente à la fois un trait de caractère individuel, mais elle reste toutefois intrinsèquement liée à l'attachement à une éthique [de service public]. L'engagement envers le Workdesign dépasse lui le cadre organisationnel, tandis que l'engagement envers les Buts publics implique à la fois des buts propres à l'organisation et d'autres plus abstraits, liés à l'action publique.

Le troisième cluster est celui des ancres d'engagement à priori publiques. II s'agit nommément des ancrages liés aux Dynamiques d'interaction sociales au travail (DISOC) ; I'Impact social et sociétal de l'action publique (ISSOC) ; I'Innovation dans le secteur public (INNOV) ; les Politiques publiques (PPUBL) ; le Service public (SPUBL) ; la gestion du Temps dans les organisations et entreprises publiques (TPSPUBL) ; ainsi que les Usagers/clients des services publics (USCL).

Enfin, un dernier cluster contient les ancres d'engagement communs aux trois dimensions susmentionnées (VALUES, DEV, IDC). Cet ancrage, qui pourrait constituer le "cœur" de l'engagement au travail dans le secteur public, porte à la fois sur les Valeurs dominantes du secteur public (VALUES), le Développement professionnel et personnel (DEV), et le Sens des tâches réalisées - capturé par l'ancre Identité 
Revue de Gestion et Management Public

professionnelle, de rôle et de carrière (IDC). Cet "ADN" de l'engagement au travail, comme le laisse voir la Figure 1, pourrait occuper une place centrale dans la zone de publicitude.

Nous discutons ci-après la portée théorique et les implications pratiques des ancres d'engagement au travail décrites ci-dessus.

\section{Discussion}

Plusieurs remarques peuvent être faites à ce stade : premièrement l'ancre d'engagement envers la profession (PROF) n'est pas du tout ressortie de nos entretiens. Le fait que notre échantillon soit majoritairement composé de personnes occupant des fonctions génériques, et quasiment d'aucune des professions établies du secteur public (policier, pompier, infirmière, médecin, enseignant etc.) est peutêtre la raison pour laquelle nous n'avons donc pas pu déceler chez les personnes interviewées, des formes d'engagement liées à la profession. De plus, l'ancre Conscience professionnelle (CPRO) identifiée dans cette étude n'est pas tout à fait comparable à l'engagement envers la profession. Elle se trouve en effet globalement partagée par l'ensemble de nos répondants, non professionnels, en référence à l'importance accordée au travail bien fait. Troisièmement, une ancre Développement personnel et professionnel (DEV) apparait, que l'on pourrait difficilement considérer comme ancre publique d'engagement en raison de son lien plus direct avec l'axe individuel d'engagement au travail. Elle n'est pas non plus évoquée dans la littérature parmi les ancres universelles d'engagement au travail (Aaron Cohen, 1999, 2000; Morrow, 1983). On remarque enfin, bien que de façon marginale, une ancre liée à l'équilibre Vie privée et travail (PRIV) positionnée volontairement en toute fin du tableau.

\subsection{Vers la spécification d'un engagement public au travail}

Nous revenons ci-après sur les ancres autres qu'individuelles et organisationnelles, dont la spécificité publique apparaît plus saillante de prime abord. Comme évoqué supra, cette spécificité reste à confirmer, notamment dans une étude plus extensive mobilisant une échelle de mesure validée. C'est pourquoi, après avoir exploré la dimensionnalité de l'engagement public au travail comme concept émergent à développer, nous en examinons plus techniquement la publicitude à l'aulne de 
Revue de Gestion et Management Public

critères existants (H. Rainey \& Bozeman, 2000; Hal G Rainey, 2011), qui nécessiteraient d'être adaptées aux ancres découvertes par cette étude, notamment en raison de leur nature parfois intangible.

\section{Les ancres publiques d'engagement au travail : quelle dimensionnalité ?}

Quatre dimensions se dégagent possiblement des ancres d'engagement au travail identifiées dans notre étude : le service public comme ancrage par excellence de l'engagement au travail dans le secteur public quel que soit le type d'organisation qui emploie ; un accent sur la carrière et la trajectoire individuelle à travers la profession ; l'organisation à travers tout ce qu'elle porte et représente symboliquement ; la manière particulière de travailler dans le secteur public (Workdesign), soit la marque employeur des organisations d'État (Charbonnier-Voirin, 2015; Yves Emery \& Kouadio, 2017; Goujon-Belghit, Gilson, \& Bourgain, 2015). En marge de la marque organisationnelle plus abstraite recoupant les buts et l'identité organisationnels, la marque employeur est liée de façon plus concrète à la façon d'organiser le travail et de gérer le temps (Brewster, Sparrow, \& Harris, 2005).

Nous détaillons ci-après ces différentes dimensions : tout d'abord, l'engagement public au travail semble à travers nos répondants s'inscrire dans ce qui fait la raison d'être de l'action publique, c'est-à-dire ses buts et ses logiques d'action spécifiques. Cet ancrage dans les buts et les politiques publiques vise avant tout un impact social et sociétal du service public. L'engagement public au travail s'ancre par conséquent dans l'attachement aux usagers (bénéficiaires) du service public, situés en bout de chaine. Ainsi donc, les valeurs et idéaux portés par le service public donnent un sens à l'emploi exercé, souvent selon des modalités permettant la mise sur pied de véritables innovations sociales. Ce premier ancrage fortement orienté vers le service public n'est pas sans rappeler l'abondante littérature sur la Motivation de service public (Hondeghem \& Vandenabeele, 2005; S. Kim \& Vandenabeele, 2009; J. Perry \& Wise, 1990; James L Perry \& Vandenabeele, 2015; Wouter Vandenabeele \& Hondeghem, 2005). Dans un registre plus inhabituel, les résultats de notre analyse font émerger le positionnement de l'individu comme acteur participant à la résolution, parfois de manière innovante, de problématiques publiques. Dans ce dernier cas, l'administration est parfois celle qui investit le plus dans du matériel de pointe selon les moyens qui sont les siens et l'orientation choisie par les acteurs politiques (Emery 2009). L'État est dans ce cadre un acteur de premier plan dans l'innovation sociale pour laquelle il peut compter sur l'exercice légitime de la puissance régalienne en vue de décider du modèle de société à développer. Pour ce 
Revue de Gestion et Management Public

faire, il donnera autant que possible les moyens nécessaires à son administration. Toutefois, cet ancrage autour de l'innovation publique reste surprenant en raison de l'image longtemps entretenue des organisations publiques comme réfractaires à toutes formes d'initiatives personnelles, notamment en raison d'une forte chape législative et politique (Barry Bozeman, 2000; De Araújo, 2001; Giauque et al., 2012; H. G. Rainey \& Steinbauer, 1999).

Étant donnés les liens étroits entre les concepts de motivation et d'engagement au travail, c'est sans surprise qu'une partie des ancres révélés par la présente étude s'oriente vers les buts, le service et les bénéficiaires de l'action publique. Notre étude contribue ainsi à élargir le concept de Motivation de service public, en appliquant le même raisonnement à l'engagement au travail. Au-delà de l'engagement organisationnel, l'engagement au public au travail se révèle comme une extension possible de la Motivation de service public. Les ancres d'engagement public au travail ont par ailleurs une forte orientation sociale et sociétale en fonction de l'activité exercée. Cela est non seulement lié à la proximité avec le niveau stratégique et décisionnel des politiques publiques, mais se perçoit également dans l'implication dans les prestations de service public à l'endroit d'usagers/clients (J.L. Perry, 1996; W. Vandenabeele et al., 2008). Cet attachement aux usagers du service public semble être ce qui donne un sens particulier aux rôles campés par les employés au sein de l'administration publique. Il s'agit là d'un lien social fortement recherché par nos répondants, et qui fait écho à certaines conceptualisations de l'ethos professionnel comme synthèse des valeurs institutionnelles et des motivations propres aux agents public (Isabelle Fortier, 2013; Kernaghan, 2000). L'ethos public naît ainsi des dynamiques sociales à l'œuvre dans le secteur public. II s'inscrit ainsi dans un processus culturel contribuant à donner un sens à la réalité de l'action publique (Newman, 2004).

Avec la mise en avant des buts et missions publiques se profilent, dans la tradition civique de l'administration publique (Boltanski \& Thévenot, 1991), toutes les approches qui relèvent l'importance accordée par les agents publics à l'intérêt général et à la poursuite du bien commun (Chanlat, 2003; Esteve, Urbig, van Witteloostuijn, \& Boyne, 2016; Giauque, Anderfuhren-Biget, et al., 2013), selon des valeurs d'équité et d'impartialité chères à l'action publique. En lien avec les idéaux de service public et de service au citoyen, un ancrage particulièrement important, voire fondamental pour les employés publics interrogés reste les valeurs partagées avec l'organisation et l'environnement de travail. Les travaux du Fit nous rappellent à ce propos que 
l'adéquation entre la personne et son environnement de travail est avant tout une question de congruence entre valeurs personnelles et valeurs dominantes (Kristof, 1996; Schneider, Brent, \& Goldstein, 2000). Cette adéquation peut être supplémentaire dans le cas où un individu entretenant certaines valeurs vient s'ajouter à une population partageant en majorité ces mêmes valeurs. Elle est complémentaire lorsque dans une optique de changement organisationnel, des valeurs et surtout des compétences nouvelles complètent celles existant préalablement au sein de l'organisation. Ces valeurs sectorielles expliquent, pour de nombreux auteurs se basant sur les travaux fondateurs de Rokeach (1973), les attitudes et de comportements propres aux employés publics (Lyons, Duxbury, \& Higgins, 2006; Nabatchi, 2012; Rokeach, 1973; Stoker, 2006; Van Wart, 1998; VigodaGadot \& Meiri, 2008). Elles nourrissent en plus un autre élément essentiel à l'engagement au travail : le sens attribué aux tâches et à l'emploi réalisé. En particulier, le sens du travail interroge la finalité de l'emploi exercé, parfois sa portée sociale ou sociétale, sinon son apport en termes de développement personnel ou professionnel.

On voit également poindre, dans cet ancrage de Service public, les notions d'identité professionnelle (à la fois de rôle et de carrière) dont la publicitude (c'est-à-dire le caractère public) peut être constitutive de l'identité des agents publics (Bourgault \& Van Dorpe, 2013; Rouban, 2010, 2014). Cette identité, nous la concevons ici comme composite, car elle rassemble la plupart des employés de l'État en un même corps. Même sans exister stricto-sensu ${ }^{6}$ en Suisse, ce corps des gens du public n'en constitue pas moins une forme d'identité collective partagée par la majorité des personnes que nous avons rencontrées au cours de nos entretiens. Il pourrait être assimilé à une sorte d'adéquation supplémentaire dans le sens des approches du Fit (Kristof-Brown, Zimmermann, \& Johnson, 2005; Schneider et al., 2000).

L'identité professionnelle et collective des employés publics implique des dynamiques sociales nourrissant un autre ancrage dominant de l'engagement au travail, soit la manière spécifique de travailler dans le secteur public, c'est à dire la conception et l'organisation du travail. Ainsi la complexité, la richesse et la sensibilité des tâches à

\footnotetext{
${ }^{6}$ Il n'existe pas de parcours formalisé d'accès à la fonction public Suisse. Pour cette raison, elle est qualifiée d'Administration ouverte intégrant ses ressources humaines selon le critère de la compétence. Contrairement à des pays comme la France ou l'Allemagne, l'absence de cette socialisation secondaire dans l'emploi public rend à priori difficile la formation d'une identité de corps.
} 
Revue de Gestion et Management Public

accomplir dans les administrations publiques commandent une organisation particulière qui suppose notamment un rapport différent à la valeur Temps. Par exemple, la qualité du processus décisionnel, dans les cas où des droits sont octroyés ou ôtés à des citoyens, impose de prendre ce temps nécessaire afin de rendre des décisions plus justes et équitables, en particulier dans les activités prudentielles ${ }^{7}$. Ce temps public revêt donc une importance toute particulière pour nos répondants dans le sens où il contribue, loin des clichés habituellement nourris sur le fonctionnariat, à la délivrance d'un service public de qualité. Tel que défini dans cette étude, le Temps public n'est pas sans rappeler les critiques liées à la quantification des indicateurs mesurant la performance publique (Bentein et al., 2004; Organisation de coopération et de développement économiques (Paris), 2011). En réalité, ce Temps représente l'une des ressources essentielles de l'action publique (Knoepfel \& Varone, 2008). En effet, les individus investissent beaucoup plus de temps dans leurs rôles d'agents publics pour autant que cela participe de l'utilité publique.

Comme corollaire à au Temps public, le Workdesign désigne également un climat de travail favorisant les interactions sociales. C'est-à-dire la conception et l'organisation de l'espace de travail, notamment à travers les pratiques de gestion du personnel (Hackman \& Oldham, 1980; Wright, 2004). On parle ici de dynamiques collaboratives et d'entraide, caractéristiques du travail au quotidien dans les organisations de service, qui loin d'entraver toute performativité, facilitent la réalisation des buts à la fois organisationnels et publics. Une flexibilisation bienvenue lorsqu'on connaît la complexité de certaines missions de service public (Brown, 2004; Ketkar \& Sett, 2009; Magnusson, Boccardelli, \& Börjesson, 2009), et qui participe également du support qu'apporte l'employeur public à ses employés. On l'aura sans doute remarqué, le concept de support organisationnel est ici élargi à tout type d'interaction sociale audelà de la seule relation employeur-employé (J. A. Coyle-Shapiro \& Shore, 2007; Eisenberger, Fasolo, \& Davislamastro, 1990; Rhoades \& Eisenberger, 2002). De telles dynamiques sociales, loin d'entraver toute performativité, facilitent la réalisation des buts organisationnels et publics.

Deux autres facteurs complètent la dimensionnalité de l'engagement au travail dans le secteur public. Le premier désigne un ancrage sur les carrières individuelles. Cet

\footnotetext{
${ }^{7}$ Champy (2012:80-94) mobilise le concept de prudence, forgé par Aristote, pour désigner, dans le secteur public, les activités pour lesquelles "l'application directe de savoirs scientifiques ou de routines est mise en défaut par la complexité et la singularité de la situation ou du problème à traiter."
} 
Revue de Gestion et Management Public

ancrage se retrouve par exemple dans le sens que poursuivent nos répondants à travers diverses tâches qui leur sont assignées quel que soit l'employeur. Le sens du travail interroge la finalité de l'emploi exercé, parfois sa portée sociale ou sociétale, sinon son apport en termes de développement personnel ou professionnel. Plus spécifiquement, il s'agit du type d'emploi occupé et exercé avec une conscience professionnelle facilitant in fine l'employabilité des personnels d'État. Dans le contexte post-bureaucratique qui caractérise la plupart des organisations publiques suisses aujourd'hui, la responsabilité de développer l'employabilité à partir d'un ensemble d'outils mis en place par l'organisation reste un élément essentiel du Contrat psychologique (Emery \& Huerlimann, 2004; Lemire, 2005).

Le deuxième facteur porte sur l'organisation publique dans tout ce qui la défini : c'està-dire ses buts et missions, de même que les groupes sociaux (équipe de travail, groupe de projet etc.) qu'elle abrite. Cet ancrage n'est manifestement pas le plus dominant chez les répondants rencontrés. Cela ne nous surprend qu'à moitié car en effet, des niveaux faibles d'engagement organisationnels dans le secteur public sont rapportés depuis les premières études comparatives avec le secteur privé (Danny L Balfour \& Wechsler, 1990; Danny L. Balfour \& Wechsler, 1996; Bourantas \& Papalexandris, 1992; G. Boyne, Jenkins, \& Poole, 1999; G. A. Boyne, 2002; Moon, 2000b; Zeffane, 1994). Ces travaux contribuent à relativiser la place de l'entité organisationnelle dans la formation de l'engagement au travail chez les employés publics. Ils nous poussent aussi à s'interroger sur la véritable nature de cet engagement, notamment sa publicitude (Barry Bozeman \& Moulton, 2011).

Les ancres d'engagement au travail dans le secteur public : quelle publicitude?

Même si la spécificité publique des ancres d'engagement identifiées, comme nous l'estimons en filigrane dans cette étude, reste à confirmer, force est de constater qu'aucune de ces ancres d'engagement n'a (à notre connaissance) été auparavant considérée dans la littérature pertinente sur l'engagement au travail (Herscovitch \& Meyer, 2002; H. Klein, 2016; J. P. Meyer et al., 2002a; John P Meyer, Stanley, \& Vandenberg, 2013; Morin et al., 2011). Par conséquent, jauger nos ancres d'engagement à l'aulne de leur publicitude requiert de mobiliser une littérature qui a pour l'instant essentiellement porté sur la distinction entre organisations publiques et privées. Ceci à la lumière de ses courants principaux que sont la publicitude fondamentale, générique, dimensionnelle et normative (Allison, 1987; Barry Bozeman, 2007; Barry Bozeman \& Moulton, 2011; Christensen, 2012; Haque, 2001). Ces travaux font partie d'une longue tradition de recherche comparative entre 
Revue de Gestion et Management Public

secteurs public et privé, et tentent d'expliquer entre autres l'attractivité des employeurs publics ou privés (Ritz $\&$ Waldner, 2011). Dans cette dernière optique, plusieurs critères sont venus compléter ceux basés sur l'autorité économique et politique longtemps mobilisés dans les recherches sur la publicitude organisationnelle. Ces critères complémentaires sont en substance le style de management, les finalités sous-jacentes aux activités publiques (soit l'intérêt et le bien-être collectifs), l'étendue de la base des bénéficiaires, ainsi que les valeurs orientant l'action publique (H. Rainey \& Bozeman, 2000; Hal G Rainey, 2011).

Ces critères pourraient être, pour certains, remobilisés afin d'évaluer la publicitude des ancres d'engagement. Parmi celles révélés dans la présente recherche, cinq se prêtent particulièrement bien à cet exercice : par exemple, le fait que les politiques publiques émanent exclusivement du gouvernement et des autorités politiques suffit à qualifier de publiques les ancres Politiques publiques (PPUBL) et Services publics (SPUBL). Ces politiques et services se distingue par leur large impact social tant ils impliquent un nombre important de parties prenantes, le tout en restant à l'abri de la concurrence du marché (Antonsen \& Jørgensen, 1997; Haque, 2001; H. Rainey, 2009). D’autre part, les finalités du service public, en tant que service à la population (ISSOC), rappellent la publicitude normative : celle qui fait de la réalisation des Valeurs publiques (VALUES) I'indice ultime de publicitude (B. Bozeman, 2002; Barry Bozeman \& Sarewitz, 2005, 2011; Christensen, 2012; Haque, 2001; Kernaghan, 2000; Van der Wal, 2008). Enfin, le rapport à la temporalité (TPSPUBL) porte lui aussi une forte empreinte publique justifiée par la nature même des tâches dans certains champs d'activités (impôts et taxes, justice, social); lesquelles nécessitent dans bien des cas une application, équitable et égalitaire des règles de droit (Buffat, 2014). La richesse de ces ancrages extra-organisationnels de l'engagement au travail, plus ou moins spécifiques au secteur public, pousse également à interroger leur possible intégration à des pratiques de gestion des ressources humaines.

\subsection{Quelles GRH pour une approche de la relation d'emploi par les ancres d'engagement}

En élargissant l'engagement public au travail à ses divers ancrages, cette étude pourrait apporter une contribution aux pratiques de gestion des ressources humaines dans le secteur public, notamment en servant de base à une individualisation des 
Revue de Gestion et Management Public

pratiques (Wisard, 2007). Nous montrons ci-après en quoi il est important d'intégrer dans une conception moderne de l'engagement au travail ses ancres multiples.

\section{L'engagement multi-ancres : une approche renouvelée de la relation au travail dans le secteur public}

Plusieurs arguments peuvent être avancés à l'appui d'un concept d'engagement au travail multi-ancres. Le premier, et le plus important, reste l'éclatement du système public en sous-groupes sociaux et domaines d'action qui représentent autant d'ancrages possibles de l'engagement au travail chez les employés publics. Ensuite, les travaux pionniers de Morrow, Reichers et Cohen ont dès le début des années 1980 contribué à conforter l'idée selon laquelle l'organisation serait un ancrage parmi d'autres de l'engagement au travail. Mieux, la définition du concept, au départ fortement axée sur l'organisation", a elle-même évolué vers l'idée d'une force "qui lie un individu à une cible sociale ou non sociale et à des actions pertinentes pour cette cible" (John P. Meyer, Becker, \& Van Dick, 2006, p. 666). Comme en réalité l'organisation n'est pas un bloc monolithique (en ceci qu'elle abrite divers sousgroupes sociaux), il serait illusoire de voir dans l'engagement organisationnel le seul lien à son emploi. Beaucoup d'employés se trouvent en effet imbriqués dans différents sous-groupes organisationnels. Ils développent potentiellement différentes formes d'attachement, d'appartenance, de loyauté et donc d'engagement envers ces groupes (Lawler, 1992).

Dans le même temps, des études plus récentes invitent à voir dans l'engagement au travail un lien psychologique se caractérisant par le dévouement volontaire et la responsabilité envers une ou plusieurs ancres, possiblement une entité (organisation ou institution), une personne, voire une idée abstraite (H. J. Klein, Molloy, \& Brinsfield, 2012). D'autre part, plusieurs travaux démontrent que l'attention aux ancres spécifiques d'engagement au travail apporte une meilleure précision à la prédiction des comportements portant sur ces mêmes ancres (Stinglhamber, Bentein, \& Vandenberghe, 2002; Stinglhamber \& Vandenberghe, 2003).

En somme, l'engagement public au travail s'inscrit dans un système relationnel multimodal envers des buts, valeurs et missions du secteur public. En mobilisant la

8 "... the relative strength of an individual's identification with and involvement in a particular organization" (Richard T. Mowday et al., 1979:226) 
Revue de Gestion et Management Public

théorie des champs de Lewin, les ancres les plus proximales (proximité physique ou psychologique) peuvent être envisagées comme celles ayant l'influence la plus prépondérante (K. Lewin, 1951; Kurt Lewin, 2008). Par exemple, si l'ancre Valeurs publiques se révèle être la plus proximale dans le profil d'engagement d'un individu, c'est à travers ses valeurs qu'on pourra mieux comprendre ses attitudes et comportements au travail (Lawler, 1992). Ainsi, l'entraide et la courtoisie constatées chez un employé public pourraient découler davantage d'un attachement à certaines valeurs que de la relation avec l'organisation à proprement parler.

Finalement, la prise en compte des ancrages multiples de l'engagement au travail dans le secteur public suppose de reconnaître les formes individuelles et empathiques que peut revêtir l'investissement au travail, à côté d'autres formes plus intrinsèquement liées à l'organisation (Paillé, 2009; Stinglhamber \& Vandenberghe, 2003). Nous analysons ci-après les implications de cette approche multi-ancres pour la gestion du capital humain.

\section{Implications pour la Gestion des ressources humaines}

S'il est vrai que l'engagement pousse l'individu à adopter des comportements en phase avec l'atteinte des buts organisationnels (Siders et al., 2001), cette performance dépend également du degré de proximité physique ou psychologique (saillance) à une ou plusieurs ancres cristallisant l'investissement individuel au travail. Avec les pratiques de gestion des ressources humaines sensibles aux ancres d'engagement des agents publics, l'organisation devient en définitive le lieu où s'expriment des profils d'engagement individuels potentiellement divers.

Sur la base des résultats de cette recherche, nous proposons cinq axes permettant d'intégrer les ancres d'engagement au travail identifiées aux pratiques de gestion des ressources humaines. Ces axes rassemblent tout d'abord des pratiques liées à la congruence Personne-organisation (selon la logique Attration-Selection-Attrition) (Schneider et al., 2000). Elles impliquent également une conception plus large de la gestion des performances (Y. Emery, Wyser, Sanchez, \& Martin, 2008), laquelle favorise une flexibilisation accrue dans la gestion opérationnelle des tâches à exécuter ainsi que les relations au travail. Elles s'inscrivent par ailleurs dans le développement d'une employabilité au service des usagers (Lemire, 2005; Véniard, 2011). Enfin, ces pratiques serviraient de base à une véritable démocratisation des relations au travail privilégiant autant que possible l'horizontalité. 
Revue de Gestion et Management Public

Ainsi donc, ces mesures pourraient contribuer à assurer la compatibilité entre les marqueurs identitaires personnels ou professionnels les plus dominants chez l'employé et ceux de l'organisation. Pour ces pratiques, les responsables $\mathrm{RH}$ pourraient s'intéresser à des ancres telles que les valeurs et l'identification à un rôle ou une profession (par ex. VALUES, IDC). Elles seraient de ce fait bienvenues dans le recrutement de personnes plus sensibles aux valeurs et aux missions publiques (Ritz, Brewer, \& Neumann, 2016). Elle pourraient également servir à mettre en place une marque employeur véritablement construite autour de l'identité organisationnelle et des finalités sous-jacente aux missions de service public (Berthon, Ewing, \& Hah, 2005; Charbonnier-Voirin, 2015).

D'autre part, la performance publique gagnerait à être conçue en intégrant toute sa complexité (Bossaert, 2005; Broeder, 1996). Ce qui nécessite de définir une véritable stratégie de Workdesign (WDSGN). C'est notamment la position adoptée par les approches contextualistes du management des performances (Pichault \& Schoenaers, 2011). Dans cette version du "Management by Objectives" adaptée à la gestion des organisations publiques, les pratiques de GRH seraient d'avantage tournées vers la promotion du travail en équipe et des modèles d'évaluation collectifs mettant en avant les résultats finaux de l'action publique. De telles pratiques trouveraient plus d'écho chez les personnes dont l'ancre d'engagement au travail est le Workdesign (J. A. M. Coyle-Shapiro \& Kessler, 2002).

Le concept de Workdesign est aussi lié à la gestion du Temps de travail. Habituellement décrié par les critiques les plus féroces de l'administration publique, la gestion du temps pourrait devenir une pièce importante d'une démarche qualité. Comme l'a montré la présente étude, les employés apprécient de disposer de suffisamment de temps pour la prise de décisions impactant substantiellement la vie de leurs usagers. Une individualisation de la gestion du temps de travail (au possible des horaires à la carte) irait dans le sens d'un tel besoin. Cette flexibilité ne repose pas uniquement sur l'autogestion, mais s'inscrit aussi dans des pratiques managériales basées sur l'équité et l'égalité de traitement (J. A. Coyle-Shapiro \& Shore, 2007; Tessema, Tsegai, Ready, Embaye, \& Windrow, 2014; Tremblay, Cloutier, Simard, Chenevert, \& Vandenberghe, 2010). On retrouve parmi ces dernières autant les mesures participant à la démocratisation des relations de travail que celles permettant une meilleure employabilité au service du citoyen (Emery \& Huerlimann, 2004; Véniard, 2011). 
Revue de Gestion et Management Public

En somme, loin de reposer uniquement sur ses dimensions "rationnelles" et instrumentales, les attentes individuelles dans la relation d'emploi sont aussi bien intrinsèques qu'intangibles. Le Tableau 1 ci-dessous revient sur les pratiques de GRH susceptibles d'être mobilisées pour autour des cinq axes structurant nos ancres d'engagement au travail.

\section{Tableau 1: Pratiques GRH à haut potentiel d'engagement au travail}

\begin{tabular}{|c|c|c|c|}
\hline ORIENTATION & ANCRES & PRATIQUES RH & REFERENCES INDICATIVES \\
\hline $\begin{array}{c}\text { FIT } \\
\text { (Personne- } \\
\text { environnement) }\end{array}$ & $\begin{array}{l}\text { VALUES } \\
\text { SPUBL } \\
\text { ISSOC } \\
\text { IDC }\end{array}$ & $\begin{array}{l}\text { Recrutement et } \\
\text { sélection basés sur } \\
\text { le Fit } \\
\text { Branding interne et } \\
\text { externe axés sur } \\
\text { l'identité et les } \\
\text { valeurs publiques }\end{array}$ & $\begin{array}{l}\text { Pfeffer and Veiga (1999) } \\
\text { Ritz and al. (2016) } \\
\text { Walton (1985) } \\
\text { Burmann et Zepelin (2005) } \\
\text { Charonnier-Voirin et al. (2014) }\end{array}$ \\
\hline $\begin{array}{c}\text { Gestion des } \\
\text { performances }\end{array}$ & $\begin{array}{l}\text { DISOC } \\
\text { WDSGN } \\
\text { TEAM }\end{array}$ & $\begin{array}{l}\text { Fonctionnement en } \\
\text { équipes de travail } \\
\text { - } \quad \begin{array}{l}\text { Gestion collective } \\
\text { des performances }\end{array} \\
\text { - } \quad \begin{array}{l}\text { Management by } \\
\text { objectives }\end{array}\end{array}$ & $\begin{array}{l}\text { Emery et Giauque (2012) } \\
\text { Gould-Williams (2004) } \\
\text { Pichault (1993) } \\
\text { Rodgers (1991) }\end{array}$ \\
\hline $\begin{array}{c}\text { Flexibilité } \\
\text { managériale et des } \\
\text { relations de travail }\end{array}$ & $\begin{array}{l}\text { WDSGN } \\
\text { TPSPUBL } \\
\text { LEAD }\end{array}$ & $\begin{array}{l}\text { - Individualisation du } \\
\text { temps de travail } \\
\text { Égalité de } \\
\text { traitement et justice } \\
\text { organisationnelle }\end{array}$ & $\begin{array}{l}\text { Fournier (2015) } \\
\text { Tremblay and al. (2010) } \\
\text { Tekleab and al. (2005) }\end{array}$ \\
\hline $\begin{array}{l}\text { Développement de } \\
\text { l'employabilité au } \\
\text { service des usagers }\end{array}$ & $\begin{array}{l}\text { CPRO } \\
\text { ISSOC } \\
\text { USCL } \\
\text { DEV }\end{array}$ & $\begin{array}{ll}\text { - } & \text { Coaching/mentorat } \\
\text { - } & \text { Orientation usagers } \\
\text { dans le management }\end{array}$ & $\begin{array}{l}\text { Allen and Eby (2008) } \\
\text { Emery (2004) } \\
\text { De Vos et al. (2011) }\end{array}$ \\
\hline $\begin{array}{c}\text { Démocratisation } \\
\text { des relations de } \\
\text { travail }\end{array}$ & $\begin{array}{l}\text { WDSGN } \\
\text { LEAD } \\
\text { DISOC }\end{array}$ & $\begin{array}{ll} & \text { Management } \\
\text { participatif } \\
\text { - } \\
\text { Leadership } \\
\text { transformationnel }\end{array}$ & $\begin{array}{l}\text { Wright et al. (2012) } \\
\text { Avolio et al. (2004) } \\
\text { Savery (1991) }\end{array}$ \\
\hline
\end{tabular}




\subsection{Limites et perspectives de recherches complémentaires}

En raison de son caractère exploratoire, la principale limite de cette étude réside dans les possibilités de généralisation de nos résultats. Les personnes interrogées, prises dans leur contexte spécifique, avec leurs histoires propres, n'épuisent de loin pas tous les cas de figure de l'administration publique suisse. Ceci malgré nos efforts pour nous assurer d'une variabilité maximale des situations, aux fins de saturation des arguments. À côté de cela, l'échantillon actuel est surreprésenté en femmes et en fonctions génériques (généralement de support) du secteur public. La surabondance de ces fonctions dans notre échantillon a probablement réduit les possibilités de découvrir des ancres d'engagement concernant particulièrement les personnes exerçant des activités situées dans le cœur de cible de l'action publique. Ce choix est parfaitement assumé : ces fonctions génériques, de par leur nature même, offrent potentiellement un point de comparaison avec des postes similaires du secteur privé, permettant ainsi de dégager une éventuelle spécificité publique de l'engagement au travail.

D'un point de vue méthodologique, la subjectivité des chercheurs a pu s'inviter dans le choix des nœuds thématiques conceptualisés ici comme ancres d'engagement au travail. Sur ce point, et en mobilisant les ancres identifiées ici, il serait intéressant de mener une enquête à plus large échelle. Celle-ci permettrait de soumettre les ancres d'engagement révélées ici à des analyses statistiques factorielles puis confirmatoires à partir d'un échantillon relativement représentatif de la fonction publique suisse. Ceci permettrait également d'assurer une validité interne et externe à notre conceptualisation de l'engagement public au travail (Churchill Jr, 1979; Nezlek, 2007; Roussel \& Wacheux, 2006; Vancouver, Millsap, \& Peters, 1994). De même, notre analyse des implications en termes de GRH, assez théorique à ce stade, pourrait être davantage systématisée dans une étape ultérieure de la présente recherche. Par exemple, d'autres chercheurs pourraient comparer le regard que portent différents profils d'engagement au travail sur les pratiques RH préconisées. Dans cette optique, l'analyse de l'impact de pratiques intégrant les ancres les plus pertinentes dans les contextes de travail publics serait intéressante.

Enfin, sur la base des ancres découvertes ici, le test d'une éventuelle publicitude, dans un autre article, pourrait commencer par la construction d'une échelle standardisée. Il faudrait pour cela définir les critères de publicitude à mobiliser. Ces critères existent 
Revue de Gestion et Management Public

actuellement dans la littérature comparant les organisations publiques à celles du secteur privé. En les élargissant, il serait tout à fait possible de les appliquer aux ancres d'engagement au travail révélées ici. L'idée d'un engagement orienté vers le service public est déjà une bonne approche. Elle pourrait en plus intégrer des critères liés aux valeurs publiques et l'éthique professionnelle des employés de l'État.

\section{Conclusion}

L'objectif de départ dans cette recherche était d'interroger les engagements au travail des agents publics œuvrant dans des environnements hybrides. Nous souhaitions en discuter ensuite la publicitude ainsi que les possibles implications en termes de GRH publique. Les dix-huit ancres d'engagement au travail révélées dans la présente recherche confirment l'intérêt d'une approche multi-ancres dans le secteur public. On retrouve parmi ces ancres d'engagement des spécificités plus ou moins publiques qui restent encore à confirmer au moyen d'une enquête quantitativement plus importante. Toutefois, la variété des ancres illustre le besoin d'une définition élargie du concept d'engagement public, et permet de relativiser, au moins conceptuellement, l'importance du niveau organisationnel de l'engagement au travail, thème largement traité dans la littérature.

Sur la base de nos résultats, nous pouvons avancer que l'engagement au travail se distingue chez les agents publics par une forte identité de rôle et de carrière, fondée sur des valeurs de service public s'exprimant dans des environnements propices à de riches développements personnels et professionnels. Les praticiens des ressources humaines dans le secteur public devraient comprendre ainsi que l'engagement des personnels est façonné par des dimensions symboliques, bien souvent portées par un système d'ancrages variés, dont l'organisation publique en tant que telle n'est qu'un aspect. Sur cette base, Certaines pratiques de gestion du capital humain, notamment en matière de performance et de carrière, gagneraient à être repensées. De façon plus générale, le thème examiné dans cette étude peut servir de base à une discussion sur la manière dont les nouveaux modes d'organisation dans le public tendent à redynamiser les rapports entre personnels et employeurs. 
Revue de Gestion et Management Public

\section{Annexe 1 : Liste des participants à l'enquête}

Tableau 2 : Liste des répondants

\begin{tabular}{|l|l|l|r|r|l|l|l|l|}
\hline Alias & Code & Genre & Age & Ancté9 $^{\text {Organisation }}$ & Fonction & Catégorie prof. & Type d'organ. \\
\hline MS & NY01 & F & 42 & 10 & Administration communale 1 & Secrétaire de direction & Support & Publique \\
\hline MCF & NY02 & F & 55 & 8 & Administration communale 1 & Cheffe de bureau & Support & Publique \\
\hline FJ & NY03 & F & 50 & 10 & Administration communale 1 & Déléguée commerciale & Spécialiste & Hybride \\
\hline MCP & NY04 & F & 30 & 5 & Administration communale 1 & Spécialiste RH & Spécialiste & Publique \\
\hline LT & NY05 & M & 29 & 5 & Administration communale 1 & Chargé de projet & Support & Publique \\
\hline FT & NY06 & M & 36 & 8 & Administration communale 1 & Électricien de réseau & Tech & Hybride \\
\hline AB & NY07 & F & 33 & 10 & Administration communale 1 & Employée d'administration & Support & Hybride \\
\hline PD & FI01 & M & 49 & 13 & Administration communale 1 & Chef de Groupe - perception & Spécialiste & Publique \\
\hline ER & FI02 & F & 38 & 10 & Administration cantonale & Secrétaire de direction & Support & Publique \\
\hline ES & FI03 & F & 38 & 15 & Administration cantonale & Secrétaire de direction & Support & Publique \\
\hline CD & UN01 & F & 50 & 20 & Université & Secrétaire administrative & Support & Hybride \\
\hline
\end{tabular}

${ }^{9}$ Ancienneté 
Revue de Gestion et Management Public

\begin{tabular}{|c|c|c|c|c|c|c|c|c|}
\hline Alias & Code & Genre & Age & Ancté $^{9}$ & Organisation & Fonction & Catégorie prof. & Type d'organ. \\
\hline SB & UN02 & $\mathrm{F}$ & 25 & 1 & Université & Assistante $\mathrm{RH}$ & Support & Hybride \\
\hline VG & UN03 & $\mathrm{F}$ & 40 & 8 & Université & HRBP & Spécialiste & Hybride \\
\hline NC & UN04 & $\mathrm{F}$ & 30 & 11 & Université & Co-RH & Support & Hybride \\
\hline LM & UN05 & $\mathrm{F}$ & 28 & 7 & Université & Conseillère RH & Support & Hybride \\
\hline $\mathrm{NZ}$ & GE01 & $\mathrm{F}$ & 45 & 8 & Administration communale 2 & Assistante Urbanisme & Spécialiste & Publique \\
\hline LA & GE02 & $\mathrm{F}$ & 47 & 13 & Administration communale 2 & Photographe & Spécialiste & Publique \\
\hline JP & GE03 & $\mathrm{F}$ & 52 & 13 & Administration communale 2 & Collaboratrice technique & Tech & Publique \\
\hline$J B$ & EM01 & $\mathrm{F}$ & 40 & 10 & Ets médico-social & Nettoyeuse & Tech & Hybride \\
\hline BX & EM02 & $\mathrm{M}$ & 40 & 10 & Ets médico-social & Aide-soignant & Tech & Hybride \\
\hline CB & EM03 & $\mathrm{F}$ & 42 & 15 & Ets médico-social & Assistante de direction & Support & Hybride \\
\hline AR & MB01 & $\mathrm{M}$ & 50 & 7 & Entreprise de transport public & Chauffeur & Tech & Publique \\
\hline EL & EC01 & $\mathrm{M}$ & 63 & 25 & Ets cantonal des assu. sociales & Collaborateur administratif & Spécialiste & Publique \\
\hline IS & ECO2 & $\mathrm{M}$ & 60 & 25 & Ets cantonal des assu. sociales & Collaborateur administratif & Spécialiste & Publique \\
\hline KM & AS01 & $\mathrm{F}$ & 48 & 18 & Dpt. fédéral des migrations & Spécialiste Asile & Spécialiste & Publique \\
\hline TT & ASO2 & $\mathrm{F}$ & 55 & 25 & Dpt. fédéral des migrations & Spécialiste asile & Spécialiste & Publique \\
\hline YZ & ASO3 & $\mathrm{F}$ & 28 & 3 & Dpt. fédéral des migrations & Spécialiste asile & Spécialiste & Publique \\
\hline JG & ASO4 & $\mathrm{M}$ & 45 & 26 & Dpt. fédéral des migrations & Spécialiste asile & Spécialiste & Publique \\
\hline
\end{tabular}


Revue de Gestion et Management Public

\begin{tabular}{|l|l|l|r|r|l|l|l|l|}
\hline Alias & Code & Genre & Age & Ancté $^{9}$ & Organisation & Fonction & Catégorie prof. & Type d'organ. \\
\hline IF & DP01 & F & 50 & 19 & Hôpital universitaire & Secrétaire de direction & Support & Hybride \\
\hline
\end{tabular}


Revue de Gestion et Management Public

\section{Annexe 2 : Tableau récapitulatif des ancres d'engagement au travail}

\begin{tabular}{|c|l|l|l|}
\hline ANCRES & CLUSTER & DEFINITION & EXTRAIT \\
\hline $\begin{array}{c}\text { BPUBL } \\
\text { (Buts publics) }\end{array}$ & $\begin{array}{l}\text { PUBL }{ }^{10}+ \\
\text { ORG }\end{array}$ & Missions et buts organisationnels & $\begin{array}{l}\text { "Personnellement je préfère m'occuper des aspects } \\
\text { stratégiques des politiques de la ville. C'est, du fait de ma } \\
\text { formation, l'expression de mon intérêt pour la politique. Je } \\
\text { trouve cela mieux que d'aligner des chiffres dans un tableau } \\
\text { Excel. (...) je pourrais me fondre assez bien dans n'importe } \\
\text { quel type d'environnement du moment que ça reste } \\
\text { stratégique et permette d'avoir une finalité sociale. (...) II } \\
\text { faut qu'il y ait un but que je partage." NY05 }\end{array}$ \\
$\begin{array}{c}\text { DISOC } \\
\text { (Dynamique et } \\
\text { interactions sociales) }\end{array}$ & PUBL & $\begin{array}{l}\text { Dynamique et interactions sociales } \\
\text { interpersonnelles caractérisées par le fait de } \\
\text { créer et de tisser du lien social entre } \\
\text { collègues }\end{array}$ & $\begin{array}{l}\text { "Je suis bien ici parce que je fais ce que j'aime. Je rencontre } \\
\text { plein de gens qui m'enrichissent. L'équipe est vraiment là, } \\
\text { on s'entend tous bien. II y a aussi la possibilité de continuer } \\
\text { à se développer, à se former, mais aussi la qualité } \\
\text { relationnelle avec les gens, l'échange." NY04 }\end{array}$ \\
\hline IDC & PUBL & $\begin{array}{l}\text { Identité professionnelle, de rôle et de } \\
\text { carrière }\end{array}$ & $\begin{array}{l}\text { "Je pense que je suis une courroie de transmission entre les } \\
\text { services et la direction." UN01 }\end{array}$ \\
\hline
\end{tabular}

${ }^{10}$ Ancre publique 
Revue de Gestion et Management Public

\begin{tabular}{|c|l|l|l|}
\hline ANCRES & CLUSTER & DEFINITION & EXTRAIT \\
\hline $\begin{array}{c}\text { (Identité } \\
\text { professionnelle, de } \\
\text { rôle et de carrière) }\end{array}$ & INNOV (Innovation \\
publique) & PUBL & $\begin{array}{l}\text { Perception du secteur public comme un } \\
\text { cadre permettant d'être à la pointe de la } \\
\text { technologie et de tester certaines } \\
\text { innovations }\end{array}$ & $\begin{array}{l}\text { "Il y a des petites choses qu'on développe dans notre } \\
\text { pratique, par exemple on ne tire pas les personnes mais on } \\
\text { les attire vers nous, qu'on partage bien dans les } \\
\text { rencontres." EM03 }\end{array}$ \\
\hline $\begin{array}{c}\text { ISSOC } \\
\text { (Impact social et } \\
\text { sociétal) }\end{array}$ & PUBL & $\begin{array}{l}\text { Impact social et sociétal de l'emploi et des } \\
\text { activités exercées }\end{array}$ & $\begin{array}{l}\text { "C'est ce qu'on devrait tous avoir en tête quand on bosse } \\
\text { pour le service public. Surtout dans une commune, on met } \\
\text { en place des prestations; on construit des infrastructures } \\
\text { dont on a le retour direct. On le voit tout de suite." NY05 }\end{array}$ \\
\hline $\begin{array}{c}\text { WDGN } \\
\text { (Workdesign - } \\
\text { organisation et } \\
\text { conception du travail) }\end{array}$ & PUBL+ \\
\hline $\begin{array}{c}\text { PPUBL } \\
\text { (Politiques publiques) }\end{array}$ & PUBL & $\begin{array}{l}\text { Logique de GRH favorisant flexibilité, } \\
\text { autonomie et la participation }\end{array}$ & $\begin{array}{l}\text { "ici le directeur nous accorde assez sa confiance je trouve. II } \\
\text { nous dit " bon cette année, j'aimerais que tu fasses ça " et } \\
\text { puis comment on y arrive-t-il s'en fiche, pourvu qu'au bout } \\
\text { de l'année on ait réussi." NY03 }\end{array}$ \\
\hline $\begin{array}{c}\text { SPUBL } \\
\text { (Service public) }\end{array}$ & PUBL & $\begin{array}{l}\text { Proximité avec le niveau décisionnel et les } \\
\text { l'activité }\end{array}$ & $\begin{array}{l}\text { "Ce qui m'intéresse c'est la notion de projet, c'est la notion } \\
\text { de développer. (...) il est important qu'il y ait une proximité } \\
\text { service au public le politique. Typiquement l'état-major." NY05 }\end{array}$ \\
\hline
\end{tabular}


Revue de Gestion et Management Public

\begin{tabular}{|c|c|c|c|}
\hline ANCRES & CLUSTER & DEFINITION & EXTRAIT \\
\hline $\begin{array}{l}\text { TPS PUBL } \\
\text { (Temps public) }\end{array}$ & PUBL & $\begin{array}{l}\text { Temps qualitatif permettant une décision } \\
\text { plus juste et équitable, notamment dans les } \\
\text { activités prudentielles }\end{array}$ & $\begin{array}{l}\text { "Le temps il est différent chez nous. On doit prendre des } \\
\text { décisions, faire des recherches. On ne doit rien laisserpasser } \\
\text { en fait. Ce temps nous permet de fournir un travail de } \\
\text { qualité. !" NY03 }\end{array}$ \\
\hline $\begin{array}{c}\text { USCL } \\
\text { (Usagers-clients) }\end{array}$ & PUBL & Usagers ou clients selon les cas & $\begin{array}{l}\text { "Mais toujours garder ce contact avec les gens, qui est } \\
\text { essentiel pour la bonne marche de l'administration. Je } \\
\text { pense que ce contact est vraiment utile (...). Je n'aimerais } \\
\text { pas que tout devienne informatisé, et que les gens passent } \\
\text { par internet pour tout faire." FI02 }\end{array}$ \\
\hline $\begin{array}{c}\text { VALUE } \\
\text { (Valeurs publiques) }\end{array}$ & PUBL & $\begin{array}{l}\text { Convergence entre valeurs personnelles et } \\
\text { organisationnelles qui guident l'action ("fit") }\end{array}$ & $\begin{array}{l}\text { "Je ne travaillerais pas par exemple pour une société qui fait } \\
\text { du pétrole. Tout simplement parce que ma personnalité, } \\
\text { mon aspect personnel est puissant dans la manière dont je } \\
\text { suis au travail. Je veux parler de mes valeurs." GE01 }\end{array}$ \\
\hline $\begin{array}{l}\text { SSTABIL } \\
\text { (Sécurité et stabilité } \\
\text { de l'emploi) }\end{array}$ & $\begin{array}{l}\text { IND + } \\
\text { PUBL }\end{array}$ & & $\begin{array}{l}\text { Je me dis que c'est une chance d'avoir un travail de } \\
\text { photographe salarié. Je sais que si je ne travaillais pas ici, je } \\
\text { ne sais pas si j'aurais la possibilité de vivre en tant } \\
\text { qu'indépendante. Après il faut aussi se vendre et j'avoue } \\
\text { que je n'ai pas ce caractère-là (...) être indépendant c'est } \\
\text { aussi prendre des risques, par rapport à la clientèle, à } \\
\text { l'assurance de retraite etc. C'est pour cela que l'aspect } \\
\text { sécurité ici à la ville (ou un autre travail) est quand même } \\
\text { important." DCA01 }\end{array}$ \\
\hline
\end{tabular}


Revue de Gestion et Management Public

\begin{tabular}{|c|c|c|c|}
\hline ANCRES & CLUSTER & DEFINITION & EXTRAIT \\
\hline $\begin{array}{l}\text { DEV } \\
\text { (Développement } \\
\text { personnel et } \\
\text { professionnel) }\end{array}$ & $\begin{array}{l}\text { PUBL + } \\
\text { IND + } \\
\text { ORG }\end{array}$ & $\begin{array}{l}\text { Développement personnel et professionnel } \\
\text { permettant un épanouissement dans son } \\
\text { travail }\end{array}$ & $\begin{array}{l}\text { "J'aime particulièrement les formations. Elles portent } \\
\text { souvent sur la manière de gérer les cas les plus compliqués } \\
\text { comme les patients d'Alzheimer, les plus agités ou stressés. } \\
\text { Ce qui compte c'est quand même d'être performant. II faut } \\
\text { savoir faire au mieux." EM03 }\end{array}$ \\
\hline $\begin{array}{c}\text { CPRO } \\
\text { (Conscience } \\
\text { professionnelle) }\end{array}$ & $\begin{array}{l}\text { IND }^{11}+ \\
\text { PUBL }\end{array}$ & $\begin{array}{l}\text { Conscience professionnelle et amour du } \\
\text { travail bien fait }\end{array}$ & $\begin{array}{l}\text { "La positivité, l'empathie sont des valeurs personnelles que } \\
\text { je retrouve dans mon organisation. Mais sans hésiter la plus } \\
\text { importante reste le professionnalisme, sans hésiter. Ça veut } \\
\text { dire bien faire son boulot, avec respect. Par exemple moi je } \\
\text { n'arrive pas à partir en sachant qu'il y a encore des choses } \\
\text { à faire; que le travail n'est pas encore terminé. Il y en a qu } \\
\text { partent sans problème ! sans état d'âme. C'est du je-m'en } \\
\text { foutisme." EM03 }\end{array}$ \\
\hline $\begin{array}{c}\text { TEAM } \\
\text { (L'Equipe de travail) }\end{array}$ & IND & $\begin{array}{l}\text { Équipe de travail, travail collectif, } \\
\text { collaboration entre collègues et d'autres } \\
\text { services }\end{array}$ & $\begin{array}{l}\text { "On collabore énormément avec les services de la ville : le } \\
\text { service ces eaux, les espaces verts, l'urbanisme etc. c'est } \\
\text { une façon de fonctionner, on est obligés de collaborer un } \\
\text { petit peu, tous ensembles. Si on ne s'écoute pas, ça ne va } \\
\text { pas jouer non plus." NY03 }\end{array}$ \\
\hline
\end{tabular}

${ }^{11}$ Ancre individuelle 
Revue de Gestion et Management Public

\begin{tabular}{|c|c|c|c|}
\hline ANCRES & CLUSTER & DEFINITION & EXTRAIT \\
\hline $\begin{array}{l}\text { JOB } \\
\text { (Travail dans le secteur } \\
\text { public) }\end{array}$ & IND & Emploi dans la fonction publique & $\begin{array}{l}\text { "Lorsque je parle de mon emploi [dans le secteur public], je } \\
\text { pense bien-être." GE01 }\end{array}$ \\
\hline $\begin{array}{c}\text { LEAD } \\
\text { (Leader/superviseur) }\end{array}$ & ORG $^{12}$ & $\begin{array}{l}\text { Leader, superviseur, manager ou top } \\
\text { management }\end{array}$ & $\begin{array}{l}\text { "Je pense qu'elle [ma loyauté] se porte vers ma cheffe de } \\
\text { service dans un premier temps, car c'est d'elle de qui je } \\
\text { dépends, qui me donne mes tâches et me fait confiance. En } \\
\text { deuxième loyauté envers mon municipal. On a une bonne } \\
\text { relation et lui il partage et discute les projets avec moi. Il y } \\
\text { a une vraie complicité qui s'est créée depuis trois ans, aussi } \\
\text { du fait qu'il apprécie mon travail." NY05 }\end{array}$ \\
\hline $\begin{array}{c}\text { ORG } \\
\text { (l'Organisation) }\end{array}$ & ORG & $\begin{array}{l}\text { L'organisation dans tout ce qu'elle renvoie } \\
\text { comme image }\end{array}$ & $\begin{array}{l}\text { "Moi je suis fière de travailler à xxx parce que c'est une ville } \\
\text { qui bouge, c'est une ville qui est ouverte, qui essaye d'aller } \\
\text { de l'avant, de trouver des solutions. (...) parce que même si } \\
\text { c'est long, c'est quand même une ville qui essaye } \\
\text { d'avancer." NY05 }\end{array}$ \\
\hline
\end{tabular}

${ }^{12}$ Ancre organisationnelle 
Bibliographie

\section{Article dans une revue}

Allen, N. J., \& Meyer, J. P. (1990). The Measurement and antecedents of affective, continuance and normative commitment to the organisation. Journal of Occupational Psychology, 63, 1-18.

Antonsen, M., \& Jørgensen, T. B. (1997). The 'Publicness' of Public Organizations. Public Administration, 75(2), 337-357. doi:10.1111/1467-9299.00064

Balfour, D. L., \& Wechsler, B. (1990). Organizational commitment: A reconceptualization and empirical test of public-private differences. Review of Public Personnel Administration, 10(3), 23:40.

Balfour, D. L., \& Wechsler, B. (1991). Commitment, performance, and productivity in public organizations. Public Productivity \& Management Review, 355-367.

Balfour, D. L., \& Wechsler, B. (1996). New approches to organizational commitment. Public Productivity \& Management Review, 19(3), 253255.

Barling, J., Wade, B., \& Fullagar, C. (1990). Predicting employee commitment to company and union: Divergent models. Journal of Occupational Psychology, 63, 49-61.

Becker, H. S. (1960). Notes on the concept of commitment. American Journal of Sociology, 66, 32-40.

Becker, T. E. (1992). Foci and bases of commitment: are they distinctions worth making? Academy of Management Journal, 35(1), 232-244.

Becker, T. E., Billings, R. S., Eveleth, D. M., \& Gilbert, N. L. (1996). Foci and bases of employee commitment: Implications for job performance. Academy of Management Journal, 39(2), 464-482. doi: $10.2307 / 256788$

Bentein, K., Vandenberghe, C., \& Dulac, T. (2004). Engagement organisationnel de continuité et indicateurs d'efficacité au travail Revue de gestion des Ressources Humaines, 53, 69-79. 
Berthon, P., Ewing, M., \& Hah, L. L. (2005). Captivating company: dimensions of attractiveness in employer branding. International journal of advertising, 24(2), 151-172.

Biétry, F., Creusier, J., Laroche, P., \& Camus, S. (2014). Soutiens perçus, engagements affectifs et succès de carrière subjectif: une approche en termes de profils. Management, 17(1), 20-37.

Biétry, F., \& Laroche, P. (2011). L'implication dans l'organisation, le syndicat et/ou la carrière. Revue de gestion des Ressources Humaines, 81(3), 19-38. doi:10.3917/grhu.081.0019

Bishop, J. W., \& Dow Scott, K. (2000). An examination of organizational and team commitment in a self-directed team environment. Journal of Applied Psychology, 85(3), $439 . \quad$ Retrieved from http://ovidsp.tx.ovid.com/ovftpdfs/FPDDNCDCNCLDJB00/fs046/ ovft/live/gv023/00004565/00004565-200006000-00009.pdf

Blau, G. J. (1985). The measurement and prediction of career commitment. Journal of Occupational Psychology, 58(4), 277-288.

Bourantas, D., Anagnostelis, J., Mantes, Y., \& Kefalas, A. G. (1990). Culture gap in Greek management. Organization Studies, 11(2), 261-283.

Bourantas, D., \& Papalexandris, N. (1992). Variables affecting organizational commitment: Private-versus publicly-owned organizations in Greece. Journal of Managerial Psychology, 7(1), 310.

Bourgault, J., \& Van Dorpe, K. (2013). Managerial reforms, Public Service Bargains and top civil servant identity. International Review of Administrative Sciences, 79(1), 49-70.

Boyne, G., Jenkins, G., \& Poole, M. (1999). Human resource management in the public and private sectors: An empirical comparison. Public Administration, 77(2), 407-420. doi:10.1111/1467-9299.00160

Boyne, G. A. (2002). Public and private management: What's the difference? Journal of Management Studies, 39(1), 97-122.

Bozeman, B. (2002). Public-value failure : when efficient markets may not do. Public Administration Review, 62(2), 145-161.

Bozeman, B., \& Moulton, S. (2011). Integrative publicness: A framework for public management strategy and performance. Journal of Public Administration Research and Theory, 21(suppl 3), i363-i380. 
Revue de Gestion et Management Public

Bozeman, B., \& Sarewitz, D. (2005). Public values and public failure in US science policy. Science and Public Policy, 32(2), 119-136.

Bozeman, B., \& Sarewitz, D. (2011). Public value mapping and science policy evaluation. Minerva, 49(1), 1-23.

Brewster, C., Sparrow, P., \& Harris, H. (2005). Towards a new model of globalizing HRM. The International Journal of Human Resource Management, 16(6), 949-970. doi:10.1080/09585190500120590

Brown, K. (2004). Human resource management in the public sector. Public Management Review, 6(3), 303-309.

Buchanan, B. (1974). Building Organizational Commitment - Socialization of Managers in Work Organizations. Administrative Science Quarterly, 19(4), 533-546. Retrieved from <Go to ISI>://WOS:A1974U910900007

Buffat, A. (2014). "C'est l'étiquette État, mais comme une PME ! " : Hybridation organisationnelle, sentiments d'appartenance et stratégies identitaires des collaborateurs d'une caisse publique de chômage en Suisse. Revue Internationale des Sciences Administratives, 80(1), 71-89. doi:10.3917/risa.801.0071

Castaing, S. (2006). The effects of psychological contract fulfilment and public service motivation on organizational commitment in the French civil service. Public Policy and Administration, 21(1), 84-98.

Charbonnier-Voirin, A. (2015). Marque employeur interne et externe. Revue française de gestion(1), 63-82.

Cho, K.-H., \& Lee, S.-H. (2001). Another look at public-private distinction and organizational commitment: A cultural explanation. The International Journal of Organizational Analysis, 9(1), 84-102.

Christensen, T. (2012). Post-NPM and changing public governance. Meiji Journal of Political Science and Economics, 1(1), 1-11.

Churchill Jr, G. A. (1979). A paradigm for developing better measures of marketing constructs. Journal of marketing research, 64-73.

Clugston, M., Howell, J. P., \& Dorfman, P. W. (2000). Does cultural socialization predict multiple bases and foci of commitment? Journal of Management, 26(1), 5-30.

Cohen, A. (1999). Relationships among five forms of commitment: an empirical assessment. Journal of Organizational Behavior, 20(3), 
285-308. doi:10.1002/(SICI)1099-1379(199905)20:3<285::AIDJOB887>3.0.CO;2-R

Cohen, A. (2000). The Relationship between Commitment Forms and Work Outcomes: A Comparison of Three Models. Human Relations, 53(3), 387-417. doi:10.1177/0018726700533005

Coyle-Shapiro, J. A., \& Neuman, J. H. (2004). The psychological contract and individual differences: The role of exchange and creditor ideologies. Journal of Vocational Behavior, 64(1), 150-164.

Coyle-Shapiro, J. A., \& Shore, L. M. (2007). The employee-organization relationship: Where do we go from here? Human Resource Management Review, 17(2), 166-179.

Coyle-Shapiro, J. A. M., \& Kessler, I. (2002). Contingent and non-contingent working in local government: Contrasting psychological contracts. Public Administration, 80(1), 77-101. Retrieved from <Go to ISI >://000174423700004

Coyle-Shapiro, J. A. M., \& Kessler, I. (2003). The employment relationship in the U.K. public sector: A psychological contract perspective. Journal of Public Administration Research and Theory, 13(2), 213-230.

De Araújo, J. F. F. E. (2001). Improving public service delivery: the crossroads between NPM and traditional bureaucracy. Public Administration, 79(4), 915-932.

Desmarais, C., \& Gamassou, C. E. (2014). Tous motivés par le service public? Les liens entre position hiérarchique et motivation de service public. Revue Internationale des Sciences Administratives, 80(1), 133-152.

Diefenbach, T. (2009). New Public Management in Public Sector Organizations: The Dark Sides of Managerialistic 'Enlightenment'. Public Administration, 87(4), 892-909. doi:10.1111/j.14679299.2009.01766.x

Eisenberger, R., Fasolo, P., \& Davislamastro, V. (1990). Perceived Organizational Support and Employee Diligence, Commitment, and Innovation. Journal of Applied Psychology, 75(1), 51-59. Retrieved from < Go to ISI>://WOS:A1990CN46000006 
Revue de Gestion et Management Public

Ellemers, N., de Gilder, D., \& van den Heuvel, H. (1998). Career-oriented versus team-oriented commitment and behavior at work. Journal of Applied Psychology, 83(5), 717.

Emery , Y. (2009). L'apport du management de la qualité au renouveau du modèle bureaucratique. Revue de l'Innovation, 14(3), 1-24.

Emery, Y., \& Giauque, D. (2014). The hybrid universe of public administration in the 21st century. International Review of Administrative Sciences, 80(1), 23-32. Retrieved from http://ras.sagepub.com/content/80/1/23

files/422/23.html

http://ras.sagepub.com/content/80/1/23.full.pdf

Emery, Y., \& Kouadio, A. B. (2017). Marque employeur et stratégies RH pour les employeurs publics. Le cas du bassin d'emploi Franco-ValdoGenevois. Management international/International Management/Gestiòn Internacional, 21(2).

Emery, Y., Wyser, C., Sanchez, J., \& Martin, N. (2008). Swiss public servants' $\mathrm{s}$ perception of performance in a fast changing environment. International Review of Administrative Sciences, 74(2), 307-323.

Esteve, M., Urbig, D., van Witteloostuijn, A., \& Boyne, G. (2016). Prosocial Behavior and Public Service Motivation. Public Administration Review, 76(1), 177-187. doi:10.1111/puar.12480

Fornes, S. L., \& Rocco, T. S. (2013). A typology of workplace commitment elements and antecedents affecting organizational effectiveness.

Fortier, I. (2013). Ethos public et quête de sens dans cette ère de réforme: le NMP, ses critiques et les luttes pour la reconnaissance d'une spécificité du secteur public. @ GRH(4), 157-198.

Fortier, I., \& Emery , Y. (2012). L'ethos public en tant que processus social dynamique. Pyramides, revue du CERAP, 22(2), 83-114.

Giauque, D., Anderfuhren-Biget, S., \& Varone, F. (2013). HRM Practices, Intrinsic Motivators, and Organizational Performance in the Public Sector. Public Personnel Management. doi:10.1177/0091026013487121 
Giauque, D., Resenterra, F., \& Siggen, M. (2013). Stress et satisfaction au travail des cadres intermédiaires dans les hôpitaux de Suisse romande dans un contexte de réformes. @ GRH(4), 123-155.

Giauque, D., Ritz, A., Varone, F., \& Anderfuhren-Biget, S. (2012). Resigned but Satisfied: The Negative Impact of Public Service Motivation and Red Tape on Work Satisfaction. Public Administration, 90(1), 175193.

Gioia, D. A., Corley, K. G., \& Hamilton, A. L. (2013). Seeking qualitative rigor in inductive research notes on the Gioia methodology. Organizational Research Methods, 16(1), 15-31.

Gordon, M. E., Philpot, J. W., Burt, R. E., Thompson, C. A., \& Spiller, W. E. (1980). Commitment to the union: Development of a measure and an examination of its correlates. Journal of Applied Psychology, 65(4), 479.

Goujon-Belghit, A., Gilson, A., \& Bourgain, M. (2015). Repenser les liens entre la gestion du capital humain et la marque employeur perçue en contexte de mutation organisationnelle. Gestion et management public, 3(1), 53-71.

Goulet, L. R., \& Frank, M. L. (2002). Organizational commitment across three sectors: public, non-profit and for-profit. Public Personnel Management, 31(2), 201-210.

Haque, M. S. (2001). The diminishing publicness of public service under the current mode of governance. Public Administration Review, 61(1), 65-82.

Herscovitch, L., \& Meyer, J. P. (2002). Commitment to organizational change: Extension of a three-component model. Journal of Applied Psychology, 87(3), 474-487. doi:10.1037/0021-9010.87.3.474

Hondeghem, \& Vandenabeele. (2005). Valeurs et motivations dans le service public: perspective comparative. Revue française d'administration publique, 115, 463-480.

Hood, C. (1991). A Public Management for all Seasons. Public Administration, 69(1), 3-19.

Hood, C., \& Peters, G. (2004). The middle aging of new public management: Into the age of paradox? Journal of Public Administration Research and Theory, 14(3), 267-282. 
Revue de Gestion et Management Public

Horton, S. (2006). New Public Management: its impact on public servant's identity: An introduction to this symposium. International Journal of Public Sector Management, 19(6), 533-542.

Hunt, S. D., \& Morgan, R. M. (1994). Organizational commitment: one of many commitments or key mediating construct? Academy of Management Journal, 37(6), 1568-1587. Retrieved from $<$ Go to WoS>://WOS:000077086700006

Jung, C. S., \& Ritz, A. (2014). Goal Management, Management Reform, and Affective Organizational Commitment in the Public Sector. International Public Management Journal, 17(4), 463-492. doi:10.1080/10967494.2014.958801

Kernaghan, K. (2000). The post-bureaucratic organization and public service values. International Review of Administrative Sciences, 66, 91-104.

Ketkar, S., \& Sett, P. (2009). HR flexibility and firm performance: Analysis of a multi-level causal model. The International Journal of Human Resource Management, 20(5), 1009-1038.

Kim, J.-W., \& Rowley, C. (2005). Employee commitment: A review of the background, determinants and theoretical perspectives. Asia Pacific Business Review, 11(1), 105-124.

Klein, H. (2016). Commitment in organizational contexts: Introduction to the special issue. Journal of Organizational Behavior, 37(4), 489-493.

Klein, H. J., Molloy, J. C., \& Brinsfield, C. T. (2012). Reconceptualizing workplace commitment to redress a stretched construct: Revisiting assumptions and removing confounds. Academy of Management Review, 37(1), 130-151.

Kristof-Brown, A. L., Zimmermann, R. D., \& Johnson, E. C. (2005). Consequences of Individuals' Fit at Work: A Meta-Analysis of Person-Job, Person-organization, Person-Group, and PersonSupervisor Fit. Personnel Psychology, 58(2), 281-342.

Kristof, A. L. (1996). Person-organisation fit: an integrative review of fits conceptualizations, measurement, and implications. Personnel Psychology, 49, 1-49.

Lawler, E. E. (1992). The ultimate advantage: Creating the highinvolvement organization. 
Revue de Gestion et Management Public

Lemire, L. (2005). Le nouveau contrat psychologique et le développement de l'employabilité: chose promise, chose due! Revue multidisciplinaire sur l'emploi, le syndicalisme et le travail (REMEST), 1(1), 4-21.

Lewin, K. (1943). Defining the'field at a given time.'. Psychological Review, 50(3), 292.

Locke, E. A., Latham, G. P., \& Erez, M. (1988). The determinants of goal commitment. Academy of Management Review, 13(1), 23-39.

Lyons, S. T., Duxbury, L. E., \& Higgins, C. A. (2006). A Comparison of the Values and Commitment of Private Sector, Public Sector, and Parapublic Sector Employees. Public Management Review(July/August), 605-618.

Magnusson, M., Boccardelli, P., \& Börjesson, S. (2009). Managing the Efficiency-Flexibility Tension in Innovation: Strategic and Organizational Aspects. Creativity and Innovation Management, 18(1), 2-7. doi:10.1111/j.1467-8691.2009.00506.x

Markovits, Y., Davis, A. J., Fay, D., \& Dick, R. v. (2010). The link between job satisfaction and organizational commitment: Differences between public and private sector employees. International Public Management Journal, 13(2), 177-196.

Mathieu, J. E., \& Zajac, d. (1990). A review and meta-analysis of the antecedents, correlates, and consequences of organizational commitment. Psychological Bulletin, 108, 171-194.

McGregor, D. (1960). The human side of enterprise. New York, 21(166.1960).

Meyer, J. P., \& Allen, N. J. (1991). A three-component conceptualization of organizational commitment. Human Resource Management Review, 1(1), 61-89. doi:10.1016/1053-4822(91)90011-Z

Meyer, J. P., Allen, N. J., \& Smith, C. A. (1993). Commitment to Organisations and Occupations: Extension and Test of a Three-Component Conceptualization. Journal of Applied Psychology, 78(4), 538-551.

Meyer, J. P., Allen, N. J., \& Smith, C. A. (1993). Commitment to Organizations and Occupations: Extension and Test of a Three-Component Conceptualization. Journal of Applied Psychology, 78(4), 538-551. 
Revue de Gestion et Management Public

Meyer, J. P., Becker, T. E., \& Van Dick, R. (2006). Social Identities and Commitments at Work: Toward an Integrative Model. Journal of Organizational Behavior, 27(5), 665-683.

Meyer, J. P., \& Herscovitch, L. (2001). Commitment in the workplace: Toward a general model. Human Resource Management Review, 11(3), 299-326.

Meyer, J. P., \& Herscovitch, L. (2001). Commitment in the workplace; toward a general model. Human Resource Management Review, 11, 299-326.

Meyer, J. P., Stanley, D. J., Herscovitch, L., \& Topolnytsky, L. (2002a). Affective, Continuance and Normative Commitment to the Organisation : A Meta-Analysis of Antecedents, Correlates and Consequences. Journal or Vocational Behavior, 61, 20-52.

Meyer, J. P., Stanley, D. J., Herscovitch, L., \& Topolnytsky, L. (2002b). Affective, continuance, and normative commitment to the organization: A meta-analysis of antecedents, correlates, and consequences. Journal of Vocational Behavior, 61(1), 20-52. Retrieved from < Go to ISI $>$ ://000176895600002

Meyer, J. P., Stanley, L. J., \& Vandenberg, R. J. (2013). A person-centered approach to the study of commitment. Human Resource Management Review, 23(2), 190-202.

Moon, M. J. (2000a). Organizational commitment revisited in new public management. Public Performance \& Management Review, 24(2), 177-194.

Moon, M. J. (2000b). Organizational Commitment Revisited in New Public Management: Motivation, Organizational Culture, Sector, and Managerial Level. Public Performance \& Management Review, 24(2), 177-194. doi:10.2307/3381267

Morin, A. J., Vandenberghe, C., Boudrias, J.-S., Madore, I., Morizot, J., \& Tremblay, M. (2011). Affective commitment and citizenship behaviors across multiple foci. Journal of Managerial Psychology, 26(8), 716-738.

Morrow, P. C. (1983). Concept redundancy in organizational research: The case of work commitment. Academy of Management Review, 8(3), 486-500. 
Revue de Gestion et Management Public

Morrow, P. C., \& Wirth, R. E. (1989). Work commitment among salaried professionals. Journal of Vocational Behavior, 34(1), 40-56.

Mowday, R. T., Steers, R. M., \& Porter, L. W. (1979). The measurement of organizational commitment. Journal of Vocational Behavior, 14(2), 224-247. doi:10.1016/0001-8791(79)90072-1

Nabatchi, T. (2012). Putting the "public" back in public values research: Designing participation to identify and respond to values. Public Administration Review, 72(5), 699-708.

Newman, J. (2004). Constructing accountability: Network governance and managerial agency. Public Policy and Administration, 19(4), 17-33.

Nezlek, J. B. (2007). A multilevel framework for understanding relationships among traits, states, situations and behaviours. European Journal of Personality, 21(6), 789-810.

Nyhan, R. C. (1999). Increasing Affective Organizational Commitment in Public Organizations The Key Role of Interpersonal Trust. Review of Public Personnel Administration, 19(3), 58-70.

Odom, R. Y., Boxx, W. R., \& Dunn, M. G. (1990). Organizational cultures, commitment, satisfaction, and cohesion. Public Productivity \& Management Review, 157-169.

Oh, Y., \& Park, J. (2011). New link between administrative reforms and job attitude: The role of interpersonal trust in peers as a mediator on organizational commitment. International Review of Public Administration, 16(3), 65-87.

Paillé, P. (2009). Engagement et citoyenneté en contexte organisationnel: un examen empirique sur l'apport des cibles multiples d'engagement à la prédiction des comportements de citoyenneté organisationnelle. Le travail humain, 72(2), 185-204.

Perry, J., \& Wise, L. R. (1990). The motivational basis of public service. Public Administration Review, 50(3), 367-373.

Perry, J. L. (1996). Measuring Public Service Motivation: An Assessment of Construct Reliability and Validity. Journal of Public Administration Research and Theory, 6(1), 5-23.

Perry, J. L., \& Vandenabeele, W. (2015). Public service motivation research: Achievements, challenges, and future directions. Public Administration Review, 75(5), 692-699. 
Rainey, H., \& Bozeman, B. (2000). Comparing public and private organizations: empirical research and the power of the a priori. Journal of Public Administration Research and Theory, J-Part 10(2), 447-469.

Rainey, H. G. (1989). Public management: Recent research on the political context and managerial roles, structures, and behaviors. Journal of Management, 15(2), 229-250.

Rainey, H. G. (2011). Sampling designs for analyzing publicness: Alternatives and their strengths and weaknesses. Journal of Public Administration Research and Theory, 21(suppl 3), i321-i345.

Rainey, H. G., \& Steinbauer, P. (1999). Galloping elephants: developing elements of a theory of effective government organizations. Journal of Public Administration Research and Theory, 9(1), 1-32.

Rayner, J., Lawton, A., \& Williams, H. M. (2012). Organizational citizenship behavior and the public service ethos: Whither the organization? Journal of Business Ethics, 106, 117-130.

Reichers, A. E. (1985). A Review and Reconceptualization of Organizational Commitment. Academy of Management Review, 10(3), 465-476. doi:10.5465/AMR.1985.4278960

Rhoades, L., \& Eisenberger, R. (2002). Perceived organizational support: A review of the literature. Journal of Applied Psychology, 87(4), 698714.

Riketta, M. (2002). Attitudinal organizational commitment and job performance: a meta-analysis. Journal of Organizational Behavior, 23(3), 257-266.

Riketta, M., Van Dick, R., \& Rousseau, D. M. (2006). Employee attachment in the short and long run: Antecedents and consequences of situatedand deep-structure identification. Zeitschrift für Personalpsychologie, 5(3), 85-93.

Ritz, A., Brewer, G. A., \& Neumann, O. (2016). Public service motivation: A systematic literature review and outlook. Public Administration Review.

Ritz, A., \& Waldner, C. (2011). Competing for future leaders: A study of attractiveness of public sector organizations to potential job 
applicants. Review of Public Personnel Administration, $0734371 \mathrm{X} 11408703$.

Rouban, L. (2010). L'univers axiologique des fonctionnaires. Revue française d'administration publique(132), 771-788.

Savery, L. K. (1991). Perceived and preferred styles of leadership: Influences on employee job satisfaction. Journal of Managerial Psychology, 6(1), 28-32.

Scott, C. R. (1999). The impact of physical and discursive anonymity on group members' multiple identifications during computersupported decision making. Western Journal of Communication (includes Communication Reports), 63(4), 456-487.

Siders, M. A., George, G., \& Dharwadkar, R. (2001). The relationship of internal and external commitment foci to objective job performance measures. Academy of Management Journal, 44(3), 570-579.

Steijn, B., \& Leisink, P. (2006). L'engagement organisationnel du personnel du secteur public néerlandais. Revue Internationale des Sciences Administratives, 72(2), 195-211.

Stets, J. E., \& Burke, P. J. (2000). Identity theory and social identity theory. Social Psychology Quarterly, 63(3), 224-237.

Stinglhamber, F., Bentein, K., \& Vandenberghe, C. (2002). Extension of the Three-Component Model of Commitment to Five Foci: Development of measures and substantive test. European Journal of Psychological Assessment, 18(2), 123.

Stinglhamber, F., \& Vandenberghe, C. (2003). Organizations and supervisors as sources of support and targets of commitment: A longitudinal study. Journal of Organizational Behavior, 24(3), 251270.

Stoker, G. (2006). Public Value Management. American Review of Public Administration, 36(1), 41-57.

Tessema, M. T., Tsegai, G., Ready, K., Embaye, A., \& Windrow, B. (2014). Effect of employee background on perceived organizational justice: managerial implications. International Review of Administrative Sciences, 80(2), 443-463.

Tremblay, M., Cloutier, J., Simard, G., Chenevert, D., \& Vandenberghe, C. (2010). The role of HRM practices, procedural justice, 
organizational support and trust in organizational commitment and in-role and extra-role performance. International Journal of Human Resource Management, 21(3), 405-433. doi:10.1080/09585190903549056

Valéau, P. (2007). L'engagement des entrepreneurs: des doutes au second souffle. Revue internationale PME: économie et gestion de la petite et moyenne entreprise, 20(1), 121-154.

Van der Wal, Z., De Graaf, G., Lasthuizen, K. (2008). What's valued most? Similarities and differences between the organizational values of the public and private sector. Public Administration, 86(2), 465-482.

Van Dick, R., Wagner, U., Stellmacher, J., \& Christ, O. (2004). The utility of a broader conceptualization of organizational identification: which aspects really matter? Journal of Occupational and Organizational Psychology, 77(2), 171-192.

Vancouver, J. B., Millsap, R. E., \& Peters, P. A. (1994). Multilevel analysis of organizational goal congruence. Journal of Applied Psychology, $79(5), 666$.

Vandenberghe, C. (2005). L'engagement organisationnel dans le secteur public : quelques déterminants essentiels. Telescope, 12(2), 1-10.

Véniard, A. (2011). Implication et employabilité: un engagement réciproque entre salarié et employeur à construire. Revue Management \& Avenir(49), 84.

Vigoda-Gadot, E., \& Meiri, S. (2008). New Public Management Values and Person-Organization Fit: A socio-psychologicyl Approach and Empirical Examination among Public Sector Personnel. Public Administration, 86(1), 111-131.

Wright, B. E. (2004). The Role of Work Context in Work Motivation: A Public Sector Application of Goal and Social Cognitive Theories. Journal of Public Administration Research and Theory, 14(1), 59-78.

Yang, K., \& Pandey, S. K. (2009). How do perceived political environment and administrative reform affect employee commitment? Journal of Public Administration Research and Theory, 19(2), 335-360.

Zeffane, R. (1994). Patterns of organizational commitment and perceived management style: A comparison of public and private sector employees. Human Relations, 47(8), 977-1010. 
Ouvrage

Boltanski, L., \& Thévenot, L. (1991). De la justification. Les économies de la grandeur. Paris: Gallimard.

Bozeman, B. (2000). Bureaucracy and red tape: Prentice Hall.

Bozeman, B. (2007). Public values and public interest counterbalancing economic individualism. Washington, D.C.: Georgetown University Press.

Broeder, C. d. (1996). Institutions at work : commitment and flexibility on the German and Dutch labour markets. The Hague: CPB Netherlands Bureau for Economic Policy Analysis.

Christensen, T., \& Laegreid, P. (Eds.). (2007). Transcending New Public Management. The Transformation of Public Sector Reforms. Surrey (UK)/Burlington (USA): Ashgate Publishing.

Cohen, A. (2006). Toute la fonction Ressources humaines. Paris: Dunod.

Creswell, J. W. (2008). Research Design: Qualitative, Quantitative, and Mixed Methods Approaches (3rd ed.): Sage Publications, Inc.

Emery , Y., \& Giauque, D. (2016). L'acteur et la bureaucratie au XXIe siècle. [Québec]: Presses de l'Université Laval.

Hackman, J. R., \& Oldham, G. R. (1980). Work Redesign. Reading, Mass.: Addison-

Wesley.

Hall, D. (1976). Careers in Organizations. Santa Monica: Goodwear.

Hall, D. T., \& al. (1996). The Career is Dead - Long Live the Career. A Relational Approach to Careers. San Francisco: Jossey-Bass Publishers.

Lewin, K. (1951). Field theory in social science. New York: Harper and Row.

Lewin, K. (2008). A Dynamic Theory of Personality - Selected Papers: Lightning Source Inc.

Likert, R. (1961). New Patterns of management. New York: Mc Graw Hill.

Likert, R. (1967). The human Organization. New York.

Mayo, E. (1933). The human problems of an industrial civilization. Boston.

Miles, M. B., Huberman, A. M., \& Saldana, J. (2013). Qualitative Data Analysis: A Methods Sourcebook: SAGE Publications. 
Revue de Gestion et Management Public

Mowday, R. T., Porter, L. W., \& Steers, R. (1982). Employee-Organization Linkages. N.Y.: Academic Press.

Organisation de coopération et de développement économiques (Paris). (2011). Améliorer la performance des soins de santé : comment mesurer leur qualité. Paris: OCDE [diff.:] Editions OCDE.

Pollitt, C., \& Bouckaert, G. (2009). Continuity and Change in Public Policy and Management. Cheltenham: Edward Elgar.

Pollitt, C., \& Bouckaert, G. (2011). Public Management Reform: A comparative analysis-new public management, governance, and the Neo-Weberian state: Oxford University Press.

Rainey, H. (2009). Understanding and Managing Public Organizations. San Francisco: Jossey-Bass.

Rokeach, M. (1973). The nature of human values. New York: Free Press.

Rouban, L. (2014). La fonction publique en débat. Paris.

Roussel, P., \& Wacheux, F. (2006). Management des ressources humaines : méthodes de recherche en sciences humaines et sociales. Bruxelles: De Boeck.

Strauss, A., \& Corbin, J. (1998). Basics of qualitative research : Techniques and procedures for developing Grounded Theory: Sage publications.

Van Wart, M. (1998). Changing public sector values.

\section{Chapitre d'ouvrage}

Allison, G. T. (1987). Public and Private Management : are they fundamentally alike in all unimportant Aspects ? In J. M. Shafritz \& A. C. Hyde (Eds.), Classics of Public Administration (pp. 510-529). Chicago: Dorsey Press.

Chanlat, J.-F. (2003). Le managérialisme et l'éthique du bien commun: la question de la motivation au travail dans les services publics. In $\mathrm{T}$. Duvillier, J.-L. Genard, \& A. Piraux (Eds.), La motivation au travail dans les services publics. Paris: L'Harmattan.

Emery , Y., \& Huerlimann, B. (2004). L'employabilité vue comme un processus transformationnel de l'emploi. In C. e. a. Pellois (Ed.), Bilan de compétences et mutations : l'accompagnement de la personne (pp. 21-42). Berne: Peter Lang. 
Knoepfel, P., \& Varone, F. (2008). Politiques institutionnelles régulant les ressources de acteurs des politiques substantielles: un cadre d'analyse. In P. Knoepfel \& F. Varone (Eds.), Réformes de politiques institutionnelles de gestion des ressources de l'action publique (Vol. Action publique, pp. à paraître). Lausanne: Presses polytechniques et universitaires romandes.

Schneider, B., Brent, D., \& Goldstein, H. W. (2000). Attraction-selectionattrition: Toward a person-environment psychology of organizations. In W. B. Walsh, K. H. Craik, \& R. H. Price (Eds.), Person-environment psychology: New directions and perspectives (2nd ed.) (pp. 61-85). Mahwah, NJ, US: Lawrence Erlbaum Associates Publishers.

Wisard, N. (2007). Le statut de la fonction publique en mutation : contrat de droit public et individualisation des conditions de travail. In Y. Emery \& D. Giauque (Eds.), Dilemmes de la GRH publique. Lausanne: Editions Loisirs et Pédagogie.

\section{Article de conférence}

Camilleri. (2006). Impact of personal characteristics and PSM on the performance of public sector employees in Malta. Paper presented at the EGPA Conference 2006, Milano.

Kim, S., \& Vandenabeele, W. (2009, 2009/06//undefined). A strategy for building public service motivation research internationally. Paper presented at the International Public Service Motivation Research Conference.

Pichault, F., \& Schoenaers, F. (2011). Middle management under pressure: The problematic integration of performance control systems in services of general interest Paper presented at the The 6th Organization Studies Workshop: "bringing public organization and organizing back in".

Vandenabeele, W., \& Hondeghem, P. D. A. (2005). Values and motivation in public administration: Public Service Motivation in an international comparative perspective. Paper presented at the Joint EGPA-ASPA Conference : Ethics and integrity of governance - The first transatlantic dialogue, Leuven (Belgium). 
Revue de Gestion et Management Public

Vandenabeele, W., Steijn, B., Camilleri, E., \& et Al. (2008). Comparing Public Service Motivation within various europe countries : do institutional environments make a difference ? Paper presented at the EGPA Annual Conference, Rotterdam.

\section{Rapport}

Bossaert, D. (2005). The flexibilisation of the employment status of civil servants : From life tenure to more flexible employment relations? Retrieved from Luxemburg:

Reichard, C. (2002). Evaluation de la gestion des ressources humaines axée sur la performance dans certains pays de l'OCDE. Retrieved from Paris: 